\title{
DaZ und Theater: Der dramapädagogische Ansatz zur Förderung der Bildungssprache
}

\author{
Doreen Bryant
}

\begin{abstract}
Zusammenfassung
Unter DidaktikerInnen für Deutsch als Zweitsprache (DaZ) herrscht weitgehend Konsens darüber, dass die Ursache für schulisches Scheitern nicht an mangelnden mündlichen Kommunikationsfähigkeiten in der Umgangssprache liegt. Entscheidend für den Bildungserfolg ist der Erwerb des im Unterricht verwendeten (schriftnahen) Registers, welches sich durch ein hohes Maß an Planung, Informationsverdichtung, Textkohärenz/-kohäsion und lexikalischer Präzision und Variation auszeichnet (vgl. Koch \& Oesterreicher 1994). Es ist dieses hohe Sprachregister mit all seinen Facetten, das im Fokus des Tübinger Theaterprojekts 'Stadt der Kinder' steht. Die gesamte Arbeit am Theaterstück (mit der gemeinsamen Erschließung, Verdichtung und Modifizierung von Text, dem Versprachlichen von Emotionen und Bildern, dem Schaffen imaginärer Welten durch Worte usw.) bietet hierfür ein ideales Setting. Auf der Basis von Evens Dramagrammatik wurde ein dramapädagogisches Sprachförderkonzept entwickelt und erprobt, das einerseits kindgerecht motivierend wirkt und andererseits versucht, den besonderen DaZ-Ansprüchen gerecht zu werden. Die Sprachförderarbeit lanciert eine spezifische registeranhebende Inputanreicherung und bindet Phasen der expliziten Grammatikvermittlung und der Sprachreflexion in den dramapädagogischen Prozess ein. ${ }^{1}$
\end{abstract}

\section{Einleitung}

In den letzten zehn Jahren haben die außerschulischen Sprachförderangebote für Kinder aus Zuwandererfamilien erfreulicherweise stark zugenommen. Eines der wegweisenden Projekte zur Förderung von Deutsch als Zweitsprache war das vom Max-Planck-Institut für Bildungsforschung initiierte und in Zusammenarbeit mit der Bremer Schulbehörde 2004 durchgeführte JacobsSommercamp (u.a. Stanat, Baumert \& Müller (2005)). Insgesamt nahmen 150 Kinder - zwei Drittel davon mit Migrationshintergrund - am Camp teil. Um

\footnotetext{
${ }^{1}$ Mit diesem Aufsatz möchte ich Prof. Dr. Brigitte Handwerker ganz herzlich zum 60. Geburtstag gratulieren und ihr nachträglich danken für ihre mitreißende und inspirierende Lehre, durch die ich bereits während meines Studiums neugierig wurde auf das Spannungsfeld ,Fremd-/Zweitspracherwerb - Didaktik'.
} 
die Wirksamkeit von impliziter und expliziter Sprachförderung zu überprüfen, teilte man die Kinder in entsprechende Gruppen ein. Für alle Kinder wurde nachmittags eine zweistündige Theater-AG (als implizite Sprachförderung) angeboten. Darüber hinaus erhielt eine der Gruppen am Vormittag expliziten Sprachförderunterricht, während sich die andere Gruppe auch in dieser Zeit mit dem Theaterspiel beschäftigte. Es zeigte sich, dass die Kinder, die explizite Sprachförderung erhielten, sowohl in der Grammatik als auch beim Lesen einen deutlich höheren Leistungszuwachs erzielten als die Kinder, die vormittags an der Theatergruppe teilnahmen. Allerdings war der nach dem Camp festgestellte Leistungsvorsprung im sprachstrukturellen Bereich nach drei Monaten nicht mehr signifikant (vgl. Rösch 2007: 288). Auch andere auf die Ferienzeit begrenzte Sprachförderangebote im Theaterkontext sehen sich mit dem Problem derNachhaltigkeitkonfrontiert. Die unmittelbarim Anschluss messbaren Erfolge verflüchtigen sich schon bald im Schulalltag. Die große Herausforderung besteht also darin, dramapädagogische Methoden zu entwickeln, die sowohl für Ferienprojekte als auch für den Schulalltag taugen, die motivierend wirken und die die spezifischen Sprachprobleme gezielt angehen. Als aussichtsreich erscheinen didaktische Maßnahmen, bei denen explizites Wissen genau dann vermittelt wird, wenn Kinder bemerkt haben, dass sie einer bestimmten kommunikativen Aufgabe nicht nachkommen können, dies aber unbedingt wollen und daher bereit sind, ihr sprachliches Repertoire zu erweitern. Es muss also ein ansprechender handlungsorientierter Kontext geschaffen werden, der eine sprachliche Notsituation erzeugt, in der die anvisierte Zielstruktur benötigt wird und ein Ausweichen auf Behelfsstrategien das kommunikative Ziel nicht erreichen lässt. Gemäß jüngster kognitionspsychologischer Erkenntnisse sollten die Zielstrukturen in hoher Token-Frequenz (= Vorkommenshäufigkeit eines Elements im Text) bei ausgewogener Type-Frequenz (= Anzahl verschiedener Elemente, die dem gleichen Sprachmuster folgen) angeboten werden. Durch erstere wird eine solide Ankerstruktur gelegt, letztere regt die Analogiebildung und den produktiven Gebrauch an und verhindert so die Fossilisierung einzelner Konstruktionen (u.a. Bybee 2008; Casenhiser \& Goldberg 2005; Tomasello 2003). Für das Tübinger Pfingstcamp ,Stadt der Kinder' wurde ein Konzept entwickelt, das systematische, strukturfokussierte Sprachförderung in den dramapädagogischen Prozess einbindet. Im Unterschied zum Jacobs-Camp fand die explizite Sprachvermittlung nicht in separaten Unterrichtsblöcken statt, vgl. Tabelle in Abb. 1, sondern in thematisch eingebundenen dramagrammatischen Workshops, vgl. Tabelle in Abb. 2. Inhaltlich wurden Vor- und Nachmittagsaktivitäten zusammengehalten durch das übergeordnete Ziel der Entwicklung und Aufführung eines Theaterstücks und durch die von Anfang an geschaffenen szenenbasierten Gruppenidentitäten (u.a. die Zauberer, die Agenten, die Wahrsager).

Die dramagrammatische Methode ist geradezu prädestiniert, um in einem Theatercamp Anwendung zu finden, welches sich insbesondere an Kinder mit Sprachförderbedarf richtet. Sie stellt eine handlungsorientierte, ganzheitliche Alternative zum schulartigen DaZ-Unterricht dar. Obgleich ein entsprechender 


$$
\begin{array}{ll}
\text { VORMITTAG } & \text { NACHMITTAG } \\
\text { explizite Sprachförderung: } & \text { implizite Sprachförderung: } \\
\text { DaZ-Unterricht } & \text { Theaterarbeit }
\end{array}
$$

Abbildung 1: Explizite und implizite Sprachförderung im Jacobs-Sommercamp

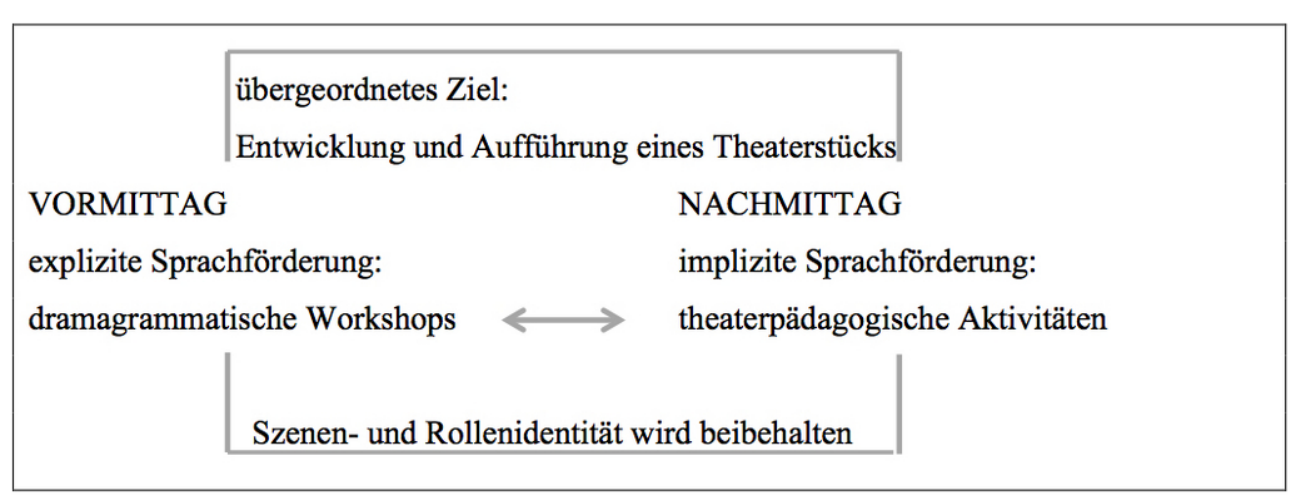

Abbildung 2: Explizite und implizite Sprachförderung im Tübinger Pfingstcamp

Nachweis noch zu erbringen ist, sehen wir in dieser von den Kindern ausgesprochen positiv angenommenen Methode große Potentiale auch im Schulalltag (sei es im Fachunterricht oder in der Theater-AG) eingesetzt zu werden, um so die Nachhaltigkeit der im Camp erzielten Lernfortschritte zu sichern und an diesen anzuknüpfen.

Der Beitrag ist folgendermaßen gegliedert. Kapitel 2 gibt zunächst einige allgemeine Informationen zum Tübinger Pfingstcamp und zur Einbettung der Sprachförderung in den Tagesablauf. In Kapitel 3 wird das Sprachförderkonzept vorgestellt. Die methodische Grundlage liefert die von Even (2003) für erwachsene DAF-Lerner entwickelte Unterrichtskonzeption der Dramagrammatik. Diese Konzeption musste einerseits an die heterogene Population der Grundschulkinder mit DaZ und deren spezifische Sprachschwierigkeiten angepasst und andererseits in ein größeres Ferienprojekt mit finaler Theateraufführung eingepasst werden. Um die Wirksamkeit der Sprachförderung zu überprüfen, wurden vor und nach dem Camp mündliche und schriftliche Sprachproben erhoben und mit denen einer Kontrollgruppe verglichen. Kapitel 4 stellt die verwendeten Evaluationsmethoden vor und geht auf einige zentrale Ergebnisse genauer ein. Folgende Hintergrundinformation ist bei der wissenschaftlichen Einordnung der Daten zu berücksichtigen: Mit der Planung und Durchführung des Tübinger Ferienprojekts bot sich universitär die Möglichkeit, ein Lehrkonzept für Studierende (angehende Deutsch-LehrerInnen und DaZ-Sprachförderkräfte) zu entwickeln und zu erproben, das zum einen Theorie und Praxis verknüpft und dabei eine Brücke zwischen Universität und 
Community schlägt und zum anderen die Studierenden in den Forschungsprozess einbindet. Das übergeordnete Ziel bestand darin, gemeinsam ein auf die spezifischen Bedürfnisse von Grundschulkindern mit DaZ zugeschnittenes dramapädagogisches Sprachförderkonzept zu entwickeln, anzuwenden, zu evaluieren und gegebenenfalls zu modifizieren. Die im Folgenden präsentierten Ergebnisse sind in diesem Ausbildungskontext entstanden und haben dementsprechend Pilotstudiencharakter. Eine unabhängige Studie zur Wirksamkeit und Nachhaltigkeit der dramagrammatischen Konzeption steht noch aus.

\section{Einbettung der Sprachförderung ins Tübinger Theaterprojekt ,Stadt der Kinder'}

Anknüpfend an die Bremer Idee (jedoch mit anders gestaltetem Sprachförderkonzept) fand in Tübingen über die Pfingstferien 2011 das 9-tägige Theaterprojekt ,Stadt der Kinder' statt, an dem insgesamt 66 Kinder mit Sprachförderbedarf teilnahmen. Etwa zwei Jahre Planung und Vorbereitung gingen dem Integrationsprojekt voraus. Kooperationspartner der Stadt Tübingen, des Landestheaters, internationaler Vereine und der Universität Tübingen erarbeiteten zusammen die Projektkonzeption, welche mit Hüttenbau, Freizeitpädagogik, Theater und Sprachförderung vier miteinander verwobene Komplexe vorsah. Ich beschränke mich im Folgenden auf meinen Verantwortungsbereich: die Sprachförderung und ihre Integration ins Gesamtkonzept. Geleistet wurde die Sprachförderung von 18 Studierenden des Deutschen Seminars der Universität Tübingen. Im Rahmen mehrerer Lehrveranstaltungen, in denen linguistische, ontogenetische, sprachdidaktische, theaterpädagogische und sozialpädagogische Inhalte vermittelt wurden, bereiteten sie sich auf die Konzipierung und Durchführung von Sprachfördereinheiten im Kontext von Theaterspiel vor. Im Verlauf der vorbereitenden Seminare entstand eine relativ umfangreiche Kartei von dramapädagogischen Sprachfördereinheiten, geordnet nach Sprachlevel, Zielstrukturen (nebst sprachtheoretisch und ontogenetisch relevanten Zusatzinformationen) und Unterrichtsphasen mit Bezugnahme auf einzelne Szenen der Geschichtsvorlage. Diese Materialsammlung erwies sich als ausgesprochen nützlicher Fundus bei den Vorbereitungen der Sprachfördereinheiten im Camp, für die täglich nur etwa zwei Stunden zur Verfügung standen. Da die Sprachförderung aufs Engste an die Theaterarbeit geknüpft war, musste die zu planende Einheit des Folgetages immer den aktuellen Stand der Improvisationen und der sich entwickelnden Szenenarbeit berücksichtigen und gleichzeitig einen Rahmen schaffen, um die angelegten sprachlichen Strukturen weiter auszubauen und zu festigen.

Unterstützt wurden die Studierenden bei ihrer Arbeit mit den Kindern von zwei Sozialpädagogen, einem Theaterpädagogen und von mir, einer Sprachwissenschaftlerin. Die drei Pädagogen und ich waren über den gesamten Zeitraum vor Ort und jederzeit ansprechbar und bereit, in schwierigen Situationen einzugreifen oder beratend zur Seite zu stehen. Darüberhinaus 
stand ich sowohl für die Reflexion der vormittäglichen Sprachfördereinheiten zur Verfügung als auch für die Planung des nächsten Tages. So die Studierenden dies wünschten, hospitierten der Theaterpädagoge oder ich in den von ihnen geleiteten Workshops. Nach diesen einleitenden Worten zu Vorbereitung und Betreuung der Studierenden soll es nun um die konkrete Realisierung der Sprachförderung gehen.

Die 18 Studierenden wurden in sechs Dreiergruppen aufgeteilt. Jedes Dreierteam übernahm die Verantwortung für 10 bis 12 Kinder. Insgesamt nahmen 66 Kinder mit Sprachförderbedarf (darunter 62 DaZ-Kinder) im Alter von 8 bis 12 Jahren am Pfingstcamp teil. Bereits im Vorfeld des Camps hatten wir von diesen Kindern Aufsätze zu einer Bildgeschichte schreiben lassen, um einen ungefähren Eindruck vom Sprachstand zu gewinnen. Am ersten Tag des Camps wurden zudem noch mündliche Sprachproben erhoben (siehe hierzu Kapitel 4). Auf der Basis der schriftlichen und mündlichen Einschätzung konnten die Kinder sechs tendenziell leistungsgleichen Gruppen zugeordnet werden, von denen zwei im oberen, zwei im mittleren und zwei im unteren Bereich lagen. Durch die Berücksichtigung des Sprachstands bei der Gruppenbildung war es uns möglich, das Sprachangebot entwicklungsproximal auf die Gruppenbedürfnisse abzustimmen.

Bereits am ersten Tag im Camp wurden die Kinder auf das übergeordnete Zielaufdiegemeinsame Erarbeitung und öffentliche Aufführung eines Theaterstücks - eingestimmt. Die noch relativ grobe Textvorlage der Geschichte wurde den Kindern unterstützt durch Standbildsequenzen von den Studierenden mittags und wiederholt am Abend vorgetragen. Auch jene Kinder, die mit Theater noch gänzlich unvertraut waren, bekamen so eine ungefähre Vorstellung vom gemeinsamen Ziel der nächsten Tage. Zudem erlebten alle Kinder die Geschichte in ihrer Gesamtheit. Dies war besonders wichtig, da jeder Gruppe die Zuständigkeit für eine Szene übertragen wurde und sich die Bedeutung und Funktion einer Szene für den Geschichtsverlauf nur im Gesamtkontext erschließt. Im Rahmen eines der vorbereitenden Seminare hatten wir sechs Schlüsselszenen identifiziert und diese je nach sprachlichen Anforderungen mit einem der drei Etiketten leicht, mittel, schwer versehen. So ist beispielsweise eine Gerichtsszene, in der verschiedene Aspekte aus verschiedenen Perspektiven vorgebracht werden müssen, sprachlich deutlich anspruchsvoller als eine Gefängnisfluchtszene. Dementsprechend wurde eine der beiden leistungsstärksten Gruppen mit der Gerichtsszene und eine der beiden schwächeren Gruppen mit der Fluchtszene betraut. Die Erarbeitung und Ausdifferenzierung einer Szene schuf den kreativen Raum für vielfältige Sprechanlässe und für eine gezielte und reflektierte Arbeit an Sprache und ihren strukturellen und lexikalischen Mitteln. Um bei der Gerichtsszene zu bleiben: Hier ließ sich die Sprachförderung mühelos koppeln an die Herausarbeitung der verschiedenen Rollen (Angeklagter, Richter, Staatsanwalt, Rechtsanwalt, Zeugen, Reporter) in Verbindung mit Improvisationen zu Status und Registern, an Wortgefechte zwischen Anwalt und Zeuge, an Argumentationen der Anwälte und des Richters, an Prozessberichte für die Zeitung usw. Zu den konkreten Sprachförderzielen 
in dieser relativ fortgeschrittenen Gruppe gehörte u.a. die Erweiterung des Spektrums an Nebensätzen und Konjunktionen. Die Kinder, die an der Gefängnisfluchtszene arbeiteten, hatten in der Sprachstandsmessung eher niedrige Werte erreicht. Sie waren aber auch jünger und stammten mehrheitlich aus der Klassenstufe 3, während sich die Gerichtsgruppe aus Kindern der Klassen 4 und 5 zusammensetzte. Im Fokus der Sprachförderung stand hier u.a. der Ausbau des Wortschatzes in der Domäne Raum. Das Fluchtthema der Szenenarbeit ließ sich ausgesprochen gut kombinieren mit der Vermittlung des komplexen Zusammenspiels von lokalen Verben (einschließlich Positions- und Bewegungsmodus), Präpositionen, Lokaladverbien und -partikeln. Der häufige Gebrauch von Partikelverben konnte zudem zur Stabilisierung der Verbklammer beitragen.

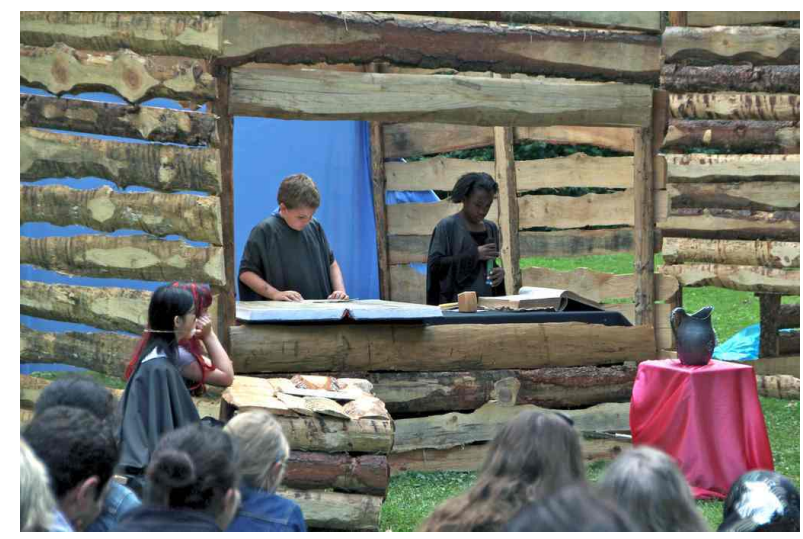

Abbildung 3: Gerichtsszene

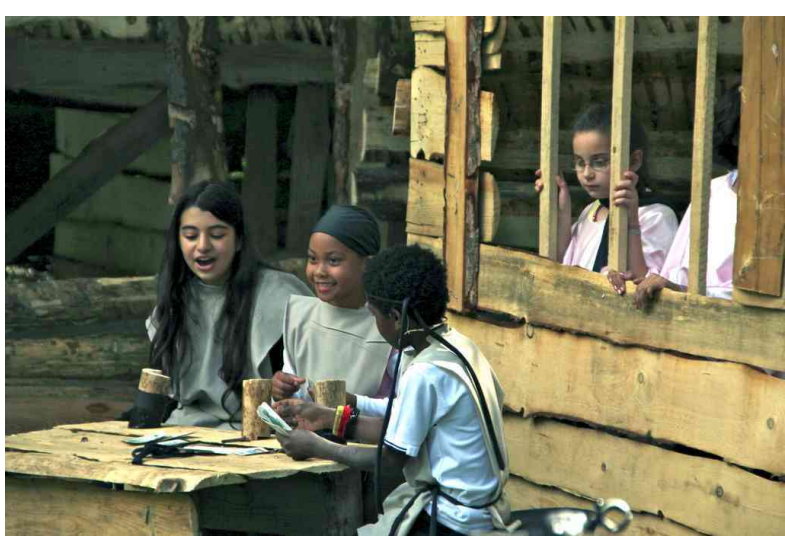

Abbildung 4: Gefängnisszene

Kommen wir nun zu ganz praktischen Fragen: Wie war der Tag strukturiert und welchen Platz nahm die Sprachförderung ein? Die Tabelle in Abb. 5 gibt hierüber Auskunft. Zwischen 8:30 und 9:00 wurden die Kinder von den Eltern gebracht und gegen 17:00 wieder abgeholt. Das Camp befand sich in einer Tübinger Grundschule mit großer Parkanlage in unmittelbarer Nachbarschaft. 
In diesem Park entstand auch das Hüttendorf - die zu bespielende Theaterkulisse. Sämtliche Verantwortlichkeiten des Tagesgeschehens (ob Essensausgabe, Planung und Durchführung von Ruhe- und Tobephasen, Hüttenbau sowie die täglichen Workshops zur Sprachförderung) lagen bei den Studierenden. Um dieser enormen Herausforderung gerecht zu werden, haben wir ein relativ komplexes Rotationssystem entwickelt. Wichtig war uns hierbei unter anderem auch, die Kommunikation zwischen den drei GruppenleiterInnen sicherzustellen. Jedes Teammitglied sollte zu jeder Zeit über den aktuellen Stand der Sprach- und Theaterarbeit in seiner Gruppe Bescheid wissen, um hierauf in seinem jeweils aktuellen Verantwortungsbereich Bezug nehmen zu können.

\begin{tabular}{|l|l|l|l|}
\hline $08: 30-09: 00$ & Frühstücksvorbereitung & A & \\
\hline $09: 00-09: 30$ & Frühstück & A & \\
\hline $09: 30-09: 45$ & gemeinsamer Auftakt & alle & \\
\hline $09: 45-12: 30$ & Workshop (WS) & B + C & \\
\hline 12:30-13:00 & Mittag & A & \\
\hline 13:00-14.00 & Ausruh-/Tobephase & C & Nachbesprechung des WS B+A \\
\hline 14:00-16:30 & Nachmittagsprogramm & A + C & Vorbereitung des WS B \\
\hline 16:30-17:00 & Abschiedsritual & alle & \\
\hline 17:00-17:30 & Aufräumen & alle & \\
\hline $17: 30-$ & Nachbesprechung & alle & \\
\hline
\end{tabular}

Abbildung 5: Tagesablauf im Camp (Rotation der Verantwortlichkeiten)

Für jede der sechs Kindergruppen war jeweils ein Dreierteam an LeiterInnen (A, B, C) zuständig. Die Arbeitsaufgaben wechselten täglich. Der Workshop für die Sprachförderung wurde immer von zwei LeiterInnen durchgeführt, wobei die Hauptverantwortung bei der Person lag, die am Tag zuvor die Planung hierfür gemacht hatte. Zwischen 13:00 und 14:00 Uhr traf sich einer der beiden Workshop-Beteiligten mit dem an diesem Tag hieran NichtBeteiligten zum Informationsaustausch. Diese Kommunikationsinsel erwies sich als ausgesprochen wichtig, da die auf den neusten Stand gebrachte Person im Nachmittagsprogramm involviert war und so in die Lage versetzt wurde, auch in den Nachmittagsaktivitäten den Bezug zu den sprachlichen Strukturen und szenischen Themen des vormittäglichen Workshops herzustellen.

Während am Vormittag die dramagrammatische Sprachförderung im Mittelpunkt stand, ging es am Nachmittag um theaterbezogene Aktivitäten wie Kulissenbau, Choreographie, Dramaturgie, Kostümherstellung. Oftmals ließ sich an die Workshop-Inhalte anknüpfen (siehe Abschnitt 3.4). Unterstützt durch szenenbezogene Gruppennamen (u.a. Zauberer, Agenten, Wahrsager), Gruppenrituale und -kleidung wurde zum einen die Gruppenidentität und die Identifizierung mit der Szene und den darin vorkommenden Charakteren gefördert und zum anderen eine Brücke zwischen Sprachförderung und Theater, 
zwischen Vormittags- und Nachmittagsangeboten geschlagen. Auch die Räumlichkeiten der Sprachförderworkshops wurden von den GruppenleiterInnen den Szenen entsprechend ausgestaltet. Die Agenten beispielsweise, die für die Geschichte der Theateraufführung eine Verratsszene zu erarbeiten hatten, trugen identitätsstiftend im Camp-Alltag alle eine dunkle Brille und ein T-Shirt mit aufgemalter Krawatte, pflegten ein geheimnisvolles Verschwörungsritual und zogen sich zum Workshop in einen Raum zurück, den die Leiterinnen labyrinthartig mit schwarzen Tüchern und Trennwänden versehen hatten. Die Wahrsager hingegen versammelten sich regelmäßig um eine Kristallkugel, in der sie die Zukunft lasen. Mit schwarzem Umhang, Basecap und Goldkette als Rapper verkleidet, prophezeiten sie dem Volke Gutes und Böses. Den Kindern dieser Gruppe konnte in der Sprachstandsmessung ein vergleichsweise hohes Sprachlevel attestiert werden. Daher kam ihnen bei der Aufführung die verantwortungsvolle Aufgabe zu, szenische Übergänge durch zusätzlich erklärende (eigene) Rap-Texte zu unterstützen.

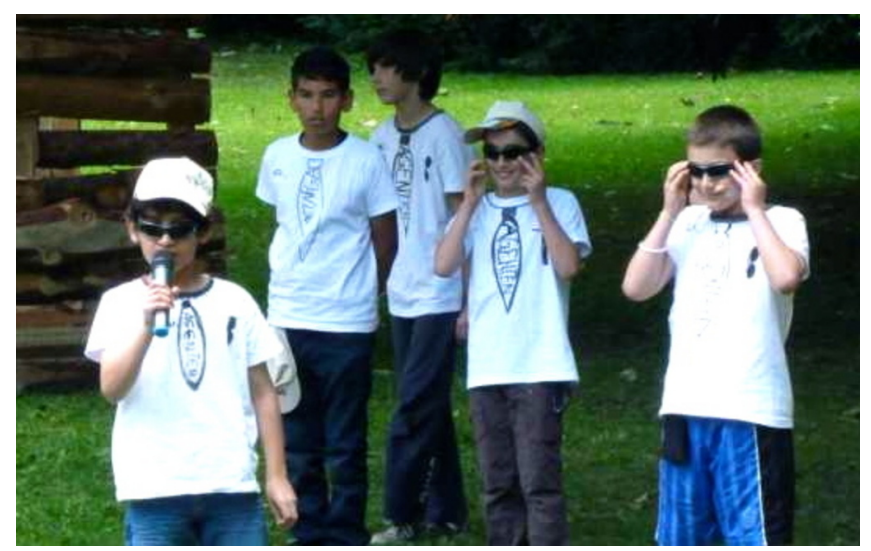

Abbildung 6: Agenten

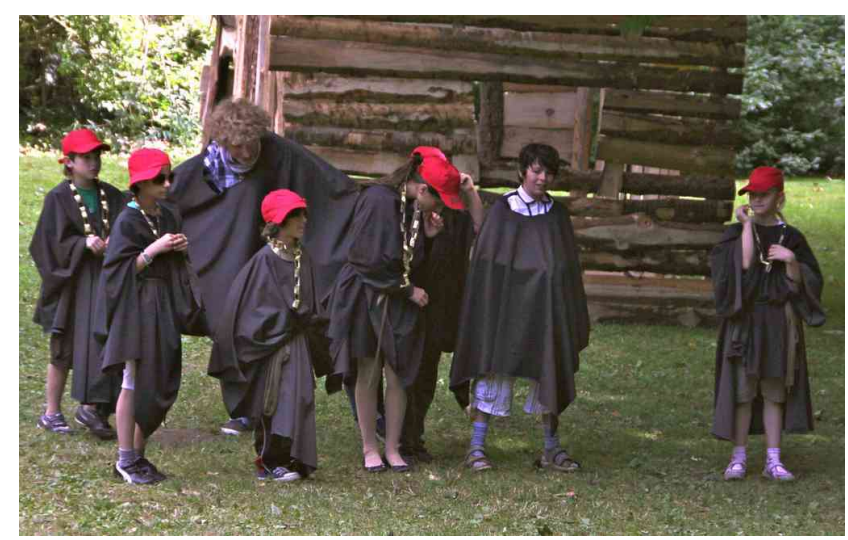

Abbildung 7: Wahrsager

Kurzum: Durch die von Anfang an geschaffenen, theaterstückbezogenen 
Identitäten gelang uns ein gleitender Übergang zwischen der expliziten Sprachförderung einerseits, die strukturfokussiert immer wieder auch szenische Themen aufgriff und den Kindern die hierfür notwendigen sprachlichen Mittel bereitstellte, und der impliziten Sprachförderung der klassischen Theaterpädagogik andererseits. Explizite und implizite Sprachförderung-üblicherweise als Kontrastpaar betrachtet - wurden im Tübinger dramapädagogischen Konzept synergetisch zusammengeführt.

\section{Das Tübinger dramapädagogische Sprachförderkonzept}

\subsection{Charakterisierung des Sprachförderkonzepts und Konkretisierung der Sprachförderziele}

Folgende Merkmale charakterisieren das Tübinger dramapädagogische Sprachförderkonzept:

- Verknüpfung expliziter und impliziter Sprachförderung;

- Einteilung der Gruppen nach Sprachstand zur entwicklungsproximalen Sprachförderung;

- Übergeordnetes Sprachförderziel: Bildungssprache (konzeptionelle Schriftlichkeit);

- Untergeordnete Sprachförderziele: diese ergeben sich aus den Merkmalen konzeptioneller Schriftlichkeit einerseits und den typischen DaZProblemfeldern andererseits, vgl. Tabelle in Abb. 10;

- Auswahl der konkreten Zielstrukturen nach entwicklungsproximalen und funktionalen Kriterien (Welche Struktur ist dem Entwicklungsstand des Kindes angemessen und lässt sich sinnvoll mit der aktuellen Szenenarbeit verknüpfen?);

- Sensibilisierung für sprachliche Register zwischen dem Nähe- und Distanzpol durch gezielte Manipulierung der Kommunikationsbedingungen, vgl. Tabelle in Abb. 8;

- Unterstützung sprachlicherTransferleistungen-konzeptionell und medial übergreifend, vgl. Abb. 9;

- Adaption des dramagrammatischen Phasenmodells von Even (2003), vgl. 3.2 ;

- Schaffen handlungsorientierter, theaterstückbezogener sprachlicher Notsituationen;

- Vorgabe des richtigen Inputs mit anschließenden Wiederholungs- und Modifizierungsübungen; 
- Einsatz verschiedener Inszenierungstechniken (insbesondere Standbildtechnik, Diashow „Augen auf, Augen zu“, Mauerschau);

- Gruppeninterne Präsentationen von szenischen Improvisationsergebnissen, Beschreibung und Interpretation des Dargestellten durch die jeweils beobachtenden Kinder;

- Korrektives Feedback: Wiederholung der Äußerung mit berichtigter Zielstruktur; Anwendung weiterer Modellierungstechniken: strukturelles Kontrastieren, Transformieren, Expandieren;

- Pre- und Posttest, breite Evaluation um eventuelle Transfereffekte feststellen zu können

(z.B. durch Schreibprobe vs. Sprechprobe, Beschreibung eines Bildes (dramapädagogisches Pendant: Standbild) vs. Beschreibung einer Bildsequenz (dramapädagogisches Pendant: Standbildsequenz, Diashow) oder freie Textproduktion vs. Strukturelizitierung (= gezieltes ,Herauslocken' bestimmter Strukturen).

Maßgebend für den Bildungserfolg ist der Erwerb der (schriftnahen) im Unterricht verwendeten Standardsprache (vgl. u.a. Siebert-Ott 2001; Gogolin \& Roth 2007; Haberzettel 2009). Man spricht auch von der sogenannten Bildungssprache. Diese weist Merkmale konzeptioneller Schriftlichkeit auf. Die Begriffe Mündlichkeit und Schriftlichkeit lassen sich zum einen in Bezug auf das Medium (Lautsprache vs. Schriftsprache) interpretieren und zum anderen in Bezug aufVersprachlichungsstrategien, die unter bestimmten Kommunikationsbedingungen der Nähe und Distanz Anwendung finden. Während das mediale Kontrastpaar auf eine Dichotomie referiert - d.h. ein sprachliches Produkt ist entweder lautsprachlich oder schriftsprachlich -, bezieht sich das zweite Kontrastpaar auf ein Kontinuum. Einelautliche Äußerung oder ein geschriebener Text lassen sich mehr oder weniger dem Nähepol bzw. dem Distanzpol zuordnen.

Die Tabelle in Abb. 8 stellt die charakteristischen Kommunikationsbedingungen des Nähe- und Distanzbereiches einander gegenüber. Während wir im Nähebreich das Register konzeptioneller Mündlichkeit gebrauchen, bedienen wir uns im Distanzbereich der sprachlichen Mittel konzeptioneller Schriftlichkeit.

Um Kinder im Erwerb der konzeptionellen Schriftlichkeit zu unterstützen, können wir dramapädagogisch die Kommunikationsbedingungen in Richtung Distanzpol verschieben und gleichzeitig den Kindern die entsprechenden sprachlichen Mittel zur Verfügung stellen. Verändert man beispielsweise (durch die Errichtung einer Trennwand) die Kommunikationsbedingung physische Nähe in physische Distanz, „zwingt“ man die Kinder (zum Beispiel bei einem improvisierten Telefongespräch) zu einer präziseren Ausdrucksweise, da der Gesprächspartner nicht der gleichen Situation beiwohnt.

Im Tübinger dramapädagogischen Sprachförderkonzept versuchen wir für die graduelle Stufung zwischen Nähe- und Distanzpol (Wer spricht wann mit 


\begin{tabular}{|l|l|}
\hline Konzeptionelle Mündlichkeit (Nähe) & Konzeptionelle Schriftlichkeit (Distanz) \\
\hline Privatheit & Öffentlichkeit \\
Vertrautheit & Fremdheit \\
Emotionalität & keine Emotionalität \\
physische Nähe & physische Distanz \\
Situations- und HandlungsEINbindung & Situations- und HandlungsENTbindung \\
Dialogizität & Monologizität \\
Spontanität & Reflektiertheit \\
\hline
\end{tabular}

Abbildung 8: Kommunikationsbedingungen für konzeptionelle Mündlichkeit / Schriftlichkeit, nach Koch \& Oesterreicher (1994)

wem auf welche Weise?) zu sensibilisieren und mit konkreten dramapädagogisch motivierten Aufgaben Transferleistungen entlang des Kontinuums zu unterstützen. Die dramapädagogischen Angebote zielen dabei insbesondere auf den konzeptionellen Transfer vom Nähe- zum Distanzpol (in Abb. 9 von links nach rechts), bei etwas älteren und fortgeschrittenen Lernern aber durchaus auch auf den medialen Transfer von der Lautsprache zur Schriftsprache (in Abb. 9 von unten nach oben). Ich werde gleich eine Sprachfördereinheit vorstellen, die zunächst einen konzeptionellen Transfer innerhalb der medialen Mündlichkeit anstrebt, um dann im zweiten Schritt die angebahnte Zielstruktur auch in die mediale Schriftlichkeit zu überführen.

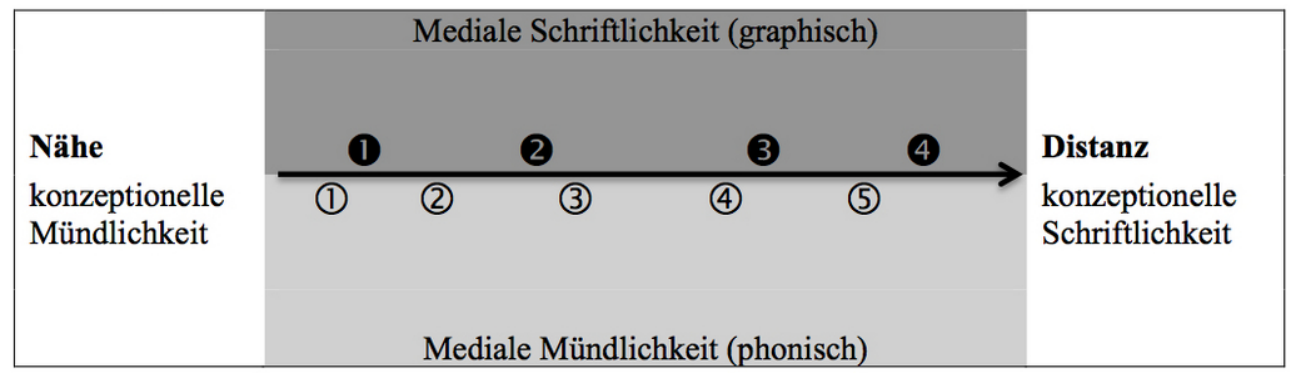

Abbildung 9: Anordnung medialer und konzeptioneller Mündlichkeit / Schriftlichkeit (in Anlehnung an Koch \& Oesterreicher 1994: 588)

In der Domäne der Lautsprache lokalisieren Koch \& Oesterreicher ganz links, dem Nähebereich zugeordnet, ein familiäres Gespräch (1), gefolgt von einem Telefongespräch (2) und ganz rechts einen wissenschaftlichen Vortrag (5). In der Domäne der Schriftsprache ordnen sie ganz links den Privatbrief (1) an (eine private Email würde man heute links davon verorten) und am äußeren 
rechten Rand Gesetzestexte (4). Um dieses Modell zu didaktisieren, muss sich die Textsortenauswahl an der Erfahrungswelt der Kinder und an den in der Schule eingeforderten Textkompetenzen orientieren. So wäre beispielsweise für Grundschulkinder der Klasse 4 ein Sachtext aus dem Schulbuch dem äußeren Distanzbereich (4) zuzuordnen. Mit der Kommunikation im Nähebereich haben DaZ-Kinder kaum Schwierigkeiten. Bei diesen sprachlichen Fähigkeiten können wir sie demnach abholen und das vorgestellte Modell für die sukzessive Erweiterung ihrer Register (via Scaffolding, vgl. u.a. Kniffka\& Siebert-Ott 2007) nutzen.

Nachdem der Begriff der konzeptionellen Schriftlichkeit eingeführt und vom Begriff der konzeptionellen Mündlichkeit abgegrenzt wurde, schauen wir nun auf die untergeordneten Sprachförderziele, die sich aus den Merkmalen konzeptioneller Schriftlichkeit einerseits und den typischen DaZ-Problemfeldern andererseits ergeben, vgl. Tabelle in Abb. 10. In Kapitel 4, in dem es um die Evaluation der Wirksamkeit der Sprachförderarbeit geht, komme ich noch einmal auf die ersten drei Sprachförderziele zurück.

\begin{tabular}{|c|c|c|c|}
\hline $\begin{array}{c}\text { Merkmale } \\
\text { konzeptioneller } \\
\text { Schriftlichkeit }\end{array}$ & & & $\begin{array}{l}\text { typische Schwierigkeiten } \\
\text { bei DAZ-Kindern }\end{array}$ \\
\hline differenzierter Wortschatz & لا & $K$ & Lokalisierungsausdrücke (a) \\
\hline \multicolumn{4}{|c|}{$\begin{array}{l}\text { Lokale Verben (Bewegungs- und Positionsmodus) } \\
\text { Lokale Präpositionen, Partikeln, Präpositionaladverbien }\end{array}$} \\
\hline Textkohäsion & لا & $\boldsymbol{K}$ & $\begin{array}{c}\text { Nominalflexion } \\
\text { (insbesondere Genus) (b) }\end{array}$ \\
\hline \multicolumn{4}{|c|}{ anaphorische Pronomen, Artikelwörter } \\
\hline Informationsverdichtung & ע & $k$ & $\begin{array}{l}\text { hypotaktischer Satzbau, } \\
\text { Nebensatzvarianz (c) }\end{array}$ \\
\hline \multicolumn{4}{|c|}{$\begin{array}{c}\text { Attributsätze, Adverbialsätze (temporal, kausal, modal, lokal, ...), Komplementsätze } \\
. .\end{array}$} \\
\hline hohes $\mathrm{Ma} ß$ an Planung & ע & $K$ & Textstrukturierung (d) \\
\hline \multicolumn{4}{|c|}{ Techniken des Beschreibens und Erzählens } \\
\hline
\end{tabular}

Abbildung 10: Sprachförderziele

Erläuterungen zur Tabelle in Abb. 10: a=für eine umfassende Darstellung von Lokalisierungsausdrücken im Erst- und Zweitspracherwerb mit konkreten didaktischen Empfehlungen für Kinder mit DaZ siehe Bryant (2012); b=siehe Wegener (1995) für eine ausführliche Behandlung der Nominalflexion als 
Lerngegenstand; $\mathrm{c}=$ Vergleichsdaten von DaZ- und DaM-Kindern zum Gebrauch hypotaktischer Strukturen findet man in Steinig et. al. (2009); d=zur Entwicklung und Förderung von Textkompetenz bei Kindern mit DaZ siehe Schmölzer-Eibinger (2011).

\subsection{Das dramagrammatische Phasenmodell}

Bevor ich anhand einer ausgewählten Sprachfördereinheit illustriere, wie wir das dramagrammatische Phasenmodell mit Grundschulkindern umsetzen, möchte ich zuvor kurz auf unsere Vorlage, auf die strukturfokussierte Unterrichtskonzeption von Even eingehen. Even (2003) inkorporiert in das dreistufige dramapädagogische Modell zur Fremdsprachvermittlung von Schewe (1993) zwei zusätzliche Komponenten, die explizite Grammatikvermittlung (Phase 3) und Sprachreflexion (Phase 5) beinhalten. Damit umfasst ihr Modell insgesamt fünf Phasen:

Die Sensibilisierungsphase führt die Teilnehmenden an bestimmte grammatische Strukturen oder Sachverhalte heran, indem zunächst ein persönlicher Anhaltspunkt gefunden wird, der das Bedürfnis bzw. die Notwendigkeit sich zu äußern hervorruft. (...) In der Kontextualisierungsphase wird der vorangegangene Impuls aufgegriffen und in einen größeren Zusammenhang gestellt. So entwerfen die Teilnehmenden (...) kurze dramatische Improvisationen, durch die die Verwendung (der Zielstruktur) im Kontext dargestellt wird. (...) Nachdem die Teilnehmenden auf bestimmte Grammatikphänomene aufmerksam geworden sind und diese bereits in kontextuellen Zusammenhängen erlebt haben, wird nun im dramatischen Prozess innegehalten, um bewusstes Nachdenken über diese Phänomene anzuregen. (Die Einordnungsphase dient der) kollaborativen Regelfindung und Systemerabeitung (...) In der Intensivierungsphase wird der dramatische Faden wieder aufgenommen. Die Lernenden, die inzwischen die Möglichkeit gehabt haben, unvertrautes oder bisher undurchschautes Material konzeptuell einzuordnen, kehren zum dramatischen Schaffensprozess zurück. Die nun folgenden Aktivitäten schließen sich thematisch an die Kontextualisierungsphase an, sodass ein inhaltlicher Zusammenhang gegeben ist und sprachliches Wissen, zusammen mit persönlichen Bildern und Vorstellungen, weiter vertieft werden kann. (...) Die Phasen der Präsentation und der Reflexion schließen die jeweilige Unterrichtssequenz ab. (ebd. 174f.)

Evens Modell ist zugeschnitten auf erwachsene Fremdsprachlerner. Bei diesen kann man sicher eine gewisse Selbstmotivation und eine grundsätzliche Bereitschaft, den didaktischen Pfaden der Lehrkraft zu folgen, voraussetzen. Dies gilt nicht unbedingt für die uns anvertrauten Grundschulkinder mit Förderbedarf, von denen einige neben den sprachlichen Problemen auch Verhaltensauffälligkeiten zeigen. Man muss sich also methodisch sehr strecken, um auch diese Kinder ins Boot zu holen. Und die Angebote müssen attraktiv genug sein, damit sie dem Boot nicht gleich wieder entspringen. Im folgenden Abschnitt wird eine Sprachfördereinheit vorgestellt, mit der dies gelungen 
ist. Die Aufmerksamkeit konnte sogar über zwei Zeitstunden hinweg gehalten werden.

Auch wenn wir weitestgehend Evens Modell folgen, gibt es doch kleinere Veränderungen - u.a. auch bei der Benennung der fünf Phasen. Wir haben uns für Bezeichnungen entschieden, die den Bezug zur Zielstruktur im dramapädagogischen Prozess klarer herausstellen:

- Aufwärmphase mit strukturiertem Input und Wiederholungsübungen in Form von Improvisationsspielen

- Motivation der Zielstruktur

- Strukturvermittlung

- Strukturanwendung

- Sprachreflexion

Es folgt nun ein konkretes Beispiel aus dem Tübinger Pfingstcamp - eine Sprachfördereinheit, die ich zusammen mit Michaela Neichel für die Gruppe der „Hammer" konzipiert habe. Dieser Gruppe wurde aufgrund des (in Relation zu den anderen Gruppen) fortgeschrittenen Sprachlevels die Szene der Gerichtsverhandlung anvertraut, die sprachlich recht hohe Anforderungen stellt. In den Workshops lag der strukturelle Fokus insbesondere auf der Erweiterung des Nebensatzspektrums und der Konjunktionen.

\subsection{Beispiel einer dramagrammatischen Sprachfördereinheit}

Vermittelt werden soll der Gebrauch der indirekten Rede (Die Richterin sagte, dass ... / Der Zeuge erzählte, dass ...) - ein sprachliches Mittel, das im Vergleich zur wörtlichen Rede auf dem in Abb. 9 skizzierten Kontinuum dem Distanzpol und der konzeptionellen Schriftlichkeit näher steht. Bei spontaner Berichterstattung gebrauchen Grundschulkinder die wörtliche Rede. Der Lerngegenstand erscheint daher geeignet, um für eine graduelle Registeranhebung zu sensibilisieren. Darüber hinaus eröffnet er die Möglichkeit, das semantische Feld der redeeinleitenden Verben (sagen, erklären, berichten, betonen, befehlen, fordern ...) zu erweitern und somit zum Ausbau des Wortschatzes beizutragen. Diese Verben verlangen ein Objekt oder einen Objektsatz, sodass wir mit der indirekten Rede auch den eingeleiteten Komplementsatz einführen und/oder festigen können. ${ }^{2}$ Die Zielstruktur soll nach Anbahnung in medialer Mündlichkeit überführt werden in mediale Schriftlichkeit.

\footnotetext{
${ }^{2}$ Ist die indirekte Rede als Komplementsatz redeeinleitenden Verben (oder Verben des Denkens, Hoffens, Glaubens) untergeordnet, dann ist „der Konjunktiv als Signal der Indirektheit (...) überflüssig.“ (DUDEN 2005: 539).
} 
Aufwärmphase (Komplementsätze im Gefühlsreplay) — Für die indirekte Rede wird die Komplementsatzstruktur benötigt. Sie ist daher Gegenstand der Aufwärmphase. Die Kinder stehen im Kreis und sind angehalten die vorgegebene Struktur (z.B. Ich habe gehört, dass der Krug gestohlen wurde.) mit emotionaler Steigerung zu wiederholen. In einem nächsten Durchgang sollen sie den vorgegebenen Satz emotional anders ausgestalten als das Kind davor - z.B. wütend und dabei kräftig mit dem Fuß stampfend, ängstlich und sich dabei nervös umschauend usw. In einem dritten Durchgang variieren die Kinder Ich habe gehört, dass .... und Ich habe gesehen, dass ... mit einer begleitenden auf Ohren bzw. Augen referierenden Geste.

Motivation der Zielstruktur -Das Telefon klingelt plötzlich. Die Chefredakteurin des Schwäbischen Tagblatts (teacher in role) ist am Apparat und berichtet aufgeregt, dass ihre Reporter alle im Einsatz seien und sie nun händeringend Reporter sucht für die in einer Stunde stattfindende Gerichtsverhandlung. Die Kinder (spielen mit und) signalisieren großes Interesse, den Job zu übernehmen. Die Workshopleiter bestärken die Kinder, machen gleichzeitig aber auch deutlich, dass hierfür eine Vorbereitung notwendig sei. Sie bieten an, den Kindern zu zeigen, wie ein Reporter Bericht erstattet. Gebraucht wird die sogenannte indirekte Rede.

Strukturvermittlung -Durch Kontrastierung von wörtlicher Rede (Er hat gesagt: „Der Krug ist zerbrochen.") und indirekter Rede (Er hat gesagt, dass der Krug zerbrochen ist.) erarbeiten sich die Kinder mit Unterstützung der Workshopleiter die charakteristischen Merkmale derindirekten Rede, die im Anschluss auf einem Poster festgehalten werden. Es wird auch darauf eingegangen, dass man in der Schriftsprache eher „er sagte“ verwenden würde, im Mündlichen hingegen „er hat gesagt". (Schriftspracheist gegenüber Sprachwandelerscheinungen wie dem sogenannten Präteritumschwund konservativer.) Nachdem die Konstruktion mit dem frequentesten redeeinleitenden Verb sagen eingeführt ist, geht es im nächsten Schritt darum, die Kinder mit anderen Vertretern dieser Verbgruppe (berichten, erzählen, befehlen, ...) vertraut zu machen, um so den produktiven Umgang mit der Konstruktion anzuregen. Die verschiedenen Verben werden auf Karteikarten geschrieben und als visuelle Stützen für die nun folgende Phase überall ausgelegt.

Strukturanwendung - Ausgestattet mit der „Reportersprache“ und mit eigenen Notizblöcken geht es nun zum Gericht. Die zwei Leiter (teacher in role) spielen drei Auszüge der Gerichtsverhandlung vor. Die Reporter hören dabei Konstruktionen der wörtlichen Rede und müssen diese ihrem Berufsstand entsprechend nun verwandeln in indirekte Rede. Jeder Reporter und jede Reporterin arbeitet konzentriert an dieser „Übersetzung“. Der innere Impuls, das Gesagte als wörtliche Rede wiederzugeben, ist, wie die beiden Reporterhefte dokumentieren, stark ausgeprägt. Wir beobachten, wie sich die Kinder nun 
auch in der medialen Schriftlichkeit die neuen Konstruktionen erarbeiten. Noch während des Schreibprozesses bemerken sie, dass ihre anfänglichen Formulierungen nicht den strukturellen Vorgaben entsprechen. Nach einer selbst initiierten Korrekturphase haben sie die Struktur "geknackt" und sind nun in der Lage, mit gewonnener Sprachbewusstheit und eigenständig rekapituliertem (zuvor vermitteltem) Strukturwissen dem Automatismus, der zur wörtlichen Rede verleitet, zu begegnen.

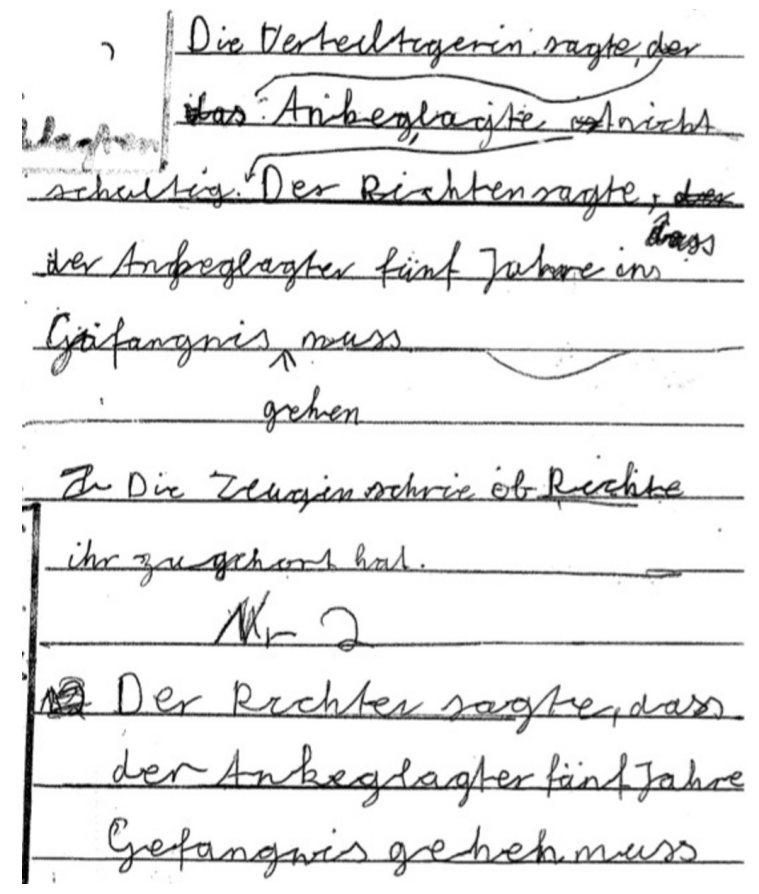

Abbildung 11: Reporternotizen von Kind A

Sprachreflexion - Die Chefredakteurin des Schwäbischen Tagblatts (teacher in role) erscheint nun und bittet die Kinder zur Redaktionssitzung. Gemeinsam wird darüber entschieden, welcher Satz eines jeden Reporters der beste ist und in der morgigen Zeitungsausgabe erscheinen soll. Es ist beeindruckend, mit welcher Ernsthaftigkeit sich die Kinder in diesem Kontext jedem einzelnen Reporterheft zuwenden und über sprachliche Strukturen reflektieren.

Mit großem Stolz stehen die Kinder am nächsten Morgen vor dem an der Wand angebrachten Zeitungsauszug (vgl. Abb. 13) und repetieren dabei erneut die Konstruktion der indirekten Rede.

\subsection{Auszug einer Szenenerarbeitung}

Als Vorlage für die Entwicklung des Theaterstücks diente eine relativ grobe Textvorlage, die der Theaterpädagoge Volker Schubert nach dem Muster einer Heldenreise verfasst hatte. Jeder Workshop-Gruppe wurde die Verantwortung 


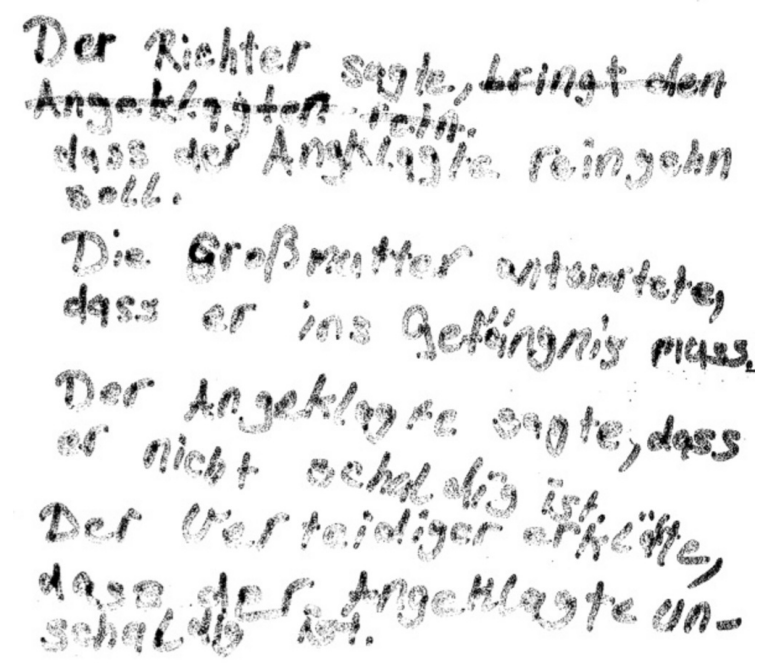

\title{
Schwäbisches Tagblatt
}

\author{
Im Gericht ist der Hammer los! \\ Harte Wortgefechte zwischen Verteidiger und Richter \\ Berichterstattung der Reporter vor Ort
}

\begin{abstract}
Tübingen, Gerichtssaal ,,der Hammer“. (Jannat) Der Richter sagte, dass der Angeklagte rein(kommen) soll. (Davide) Der Richter fragte sie, wer den Krug vergiftet hat. (Hanna) Die Großmutter antwortete, dass er ihr(en) Enkel nicht (ins) Gefängnis (schicken) darf. (Valentina) Der Angeklagte sagte, dass er nicht schuldig ist. (Zohrab) Der Verteidiger erklärte, dass er es nicht war. (Maria) Die Verteidigerin sagte, dass der
\end{abstract}

Angeklagte freigesprochen werden soll, weil er unschuldig ist. (Sharon) Der Verteidiger erklärte, dass der Schmerz vom Hunger kam. (Konstantin) Der Zeuge schwor, dass er die Wahrheit sagt. (Zainab) Die Richterin sagte, dass der Angeklagte zu 5 Jahren Gefängnis verurteilt werden muss. (Tufan) Der Verteidiger erklärte, warum er ins Gefängnis muss. (22.06.2011)

M. Neichel

Abbildung 13: Ausgewählte Sätze der dramapädagogischen Berichterstattung 
für die Erarbeitung einer Szene übertragen. Die Gruppe der Zauberer hatte die wenigsten Vorgaben und somit den größten kreativen Spielraum. In der Textvorlage hieß es lediglich: „Das Dorf ist gespalten. Ein böser Zauber hat das vor Urzeiten bewirkt. Keiner weiß mehr, warum eigentlich, aber es ist so.“

Die Aufgabe der Zauberergruppe bestand nun darin, eine Konfliktsituation entstehen zu lassen, die zur Spaltung des Dorfes führen würde. Man entschied sich einen großen Zaubererstreit zu inszenieren. In einem der dramagrammatischen Workshops ging es dementsprechend um potentielle Streitgründe. (Die hierbei eingeführten und geübten Strukturen waren u.a. Konditionalsätze folgenden Typs: Ich ärgere mich, wenn ... vs. Wenn ..., ärgere ich mich - mit unterschiedlicher Wortstellung im Hauptsatz.) Die von den Kindern genannten Ärger auslösenden Situationen wurden auf einem Poster schriftlich festgehalten. Aus diesem Fundus galt es dann gemeinschaftlich zwei Situationen auszuwählen, die von allen als besonders schlimm empfunden wurden und die daher vielleicht auch als Auslöser für den Zaubererstreit fungieren könnten. Die Kinder entschieden sich für eine Situation, in der einem etwas Kostbares weggenommen wird und für eine Situation des Ausgelachtwerdens, weil man (angeblich) verliebt sei. Die 12 Kinder teilten sich dann in zwei Gruppen, von der jede eine der beiden Situationen als Standbildsequenz vorbereitete. Im Anschluss präsentierte man sich gegenseitig die fünfteiligen Sequenzen als Diashow („Augen auf, Augen zu“), wobei der beobachtenden Gruppe die Aufgabe der Versprachlichung zukam. Am Nachmittag zeigten alle Workshop-Gruppen im ,Grünen Klassenzimmer', wo sie aktuell mit ihrer Szenenarbeit stehen. Die Zauberer stellten ihre zwei Diashows vor und das Publikum durfte entscheiden, welche der beiden Situationen für das Theaterstück weiter ausgebaut werden solle.

\section{Evaluationsmethoden und erste Ergebnisse}

Das Theatercamp wurde sowohl aus erziehungswissenschaftlicher als auch sprachwissenschaftlicher Perspektive evaluiert, vgl. Abb. 15.

Im Folgenden möchte ich auf einige zentrale Aspekte der sprachwissenschaftlichen Evaluation eingehen. Um die Wirksamkeit einer Intervention feststellen zu können, ist es erforderlich, den Sprachstand vor und nach der Maßnahme zu überprüfen und mit einer Kontrollgruppe, die im gleichen Zeitabstand ebenfalls zweimal getestet wird, zu vergleichen.

Bereits im Vorfeld des Camps wurden die schriftlichen Sprachproben (vgl. Abb. 16) erhoben. Auf der Basis der Aufsätze war es uns möglich, die Kinder ihrem Entwicklungsstand entsprechend in vier Gruppen zu unterteilen. Die hierfür zugrunde gelegten Kriterien waren: Textlänge, Wortschatzvarianz/angemessenheit, Wortstellung, Satztypen (Nebensatzvarianz), Attribute, Koreferenz, Erzählstruktur (isoliert, linear, strukturiert, narrativ strukturiert). Am ersten Tag im Camp waren vier Diagnostikteams (jeweils 2 Studierende) im Einsatz, um mit den Kindern den mündlichen Pretest, bestehend aus zwei Teilen, durchzuführen. Jedes Team untersuchte eine der vier zuvor ermittelten 


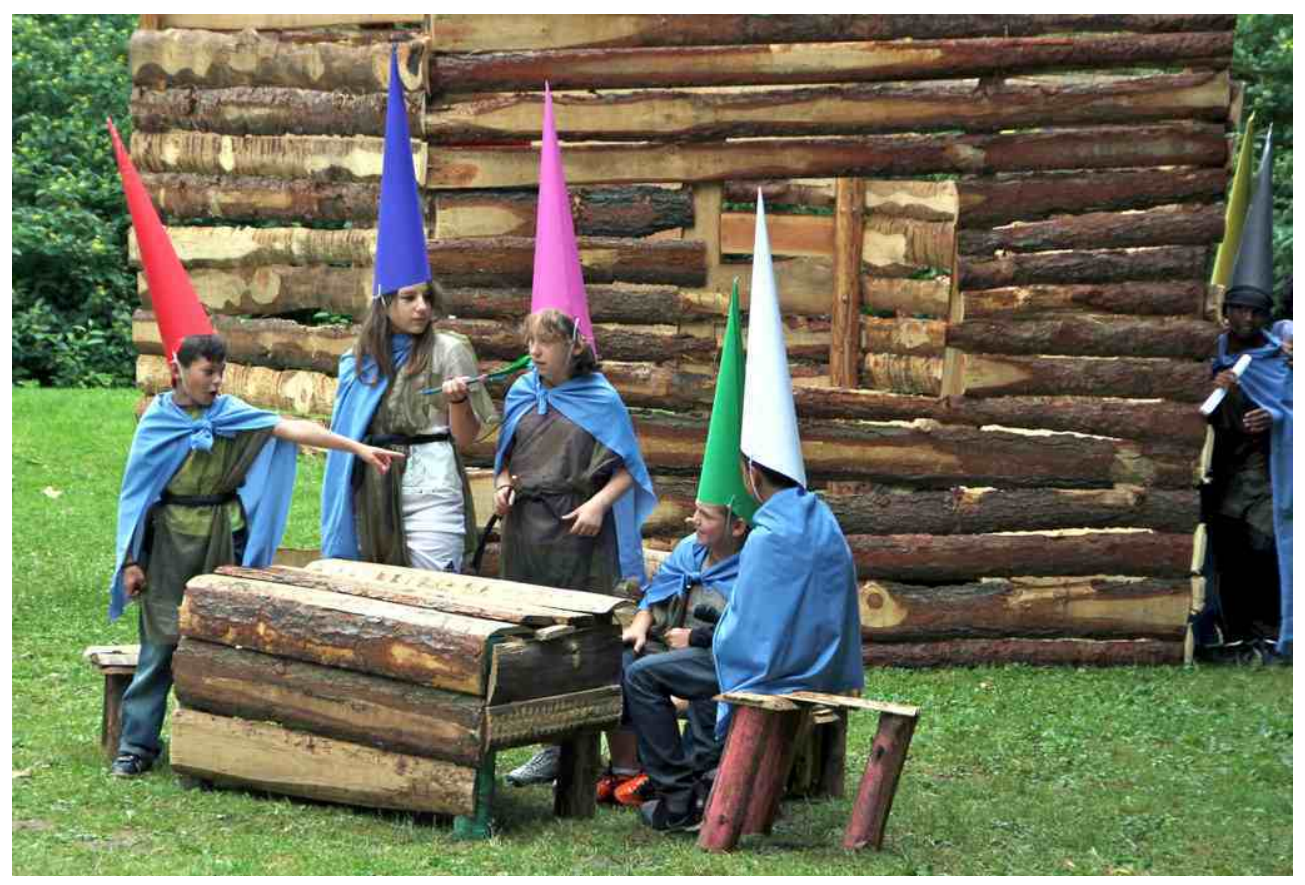

Abbildung 14: Der Zaubererstreit

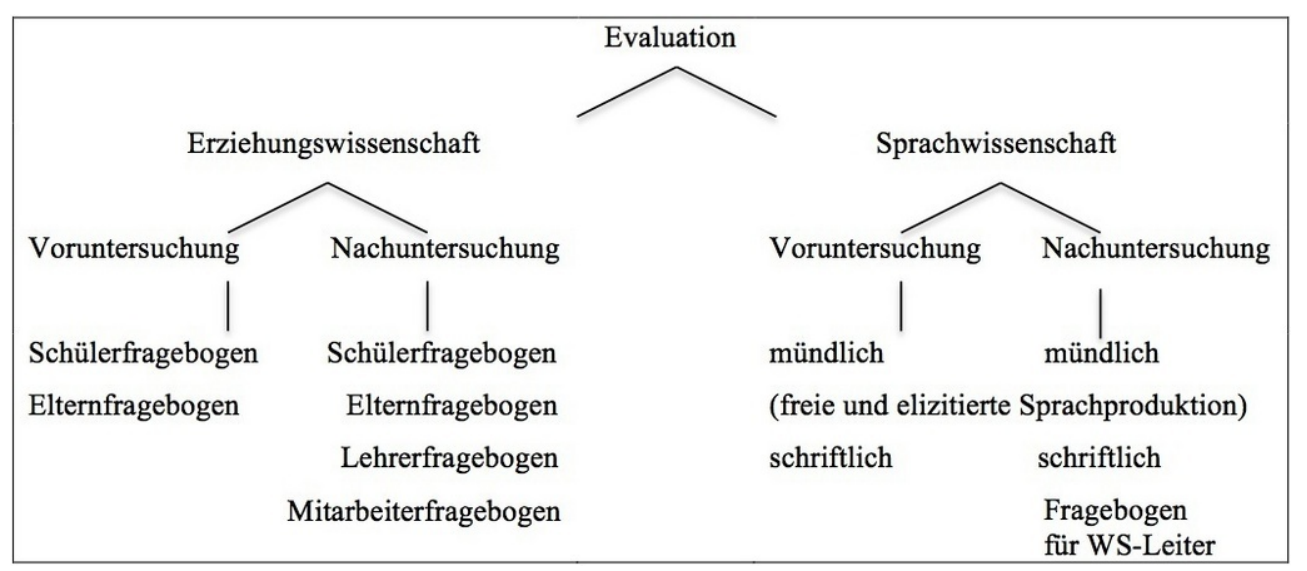

Abbildung 15: Evaluation 
Sprachstandsgruppen. So bekamen wir einen relativ differenzierten Eindruck über die sprachlichen Fähigkeiten eines jeden Kindes. Es zeigte sich, dass einige Kinder im mündlichen Bereich deutlich besser waren und daher zur Sprachförderung in eine höhere Gruppe eingestuft werden konnten.

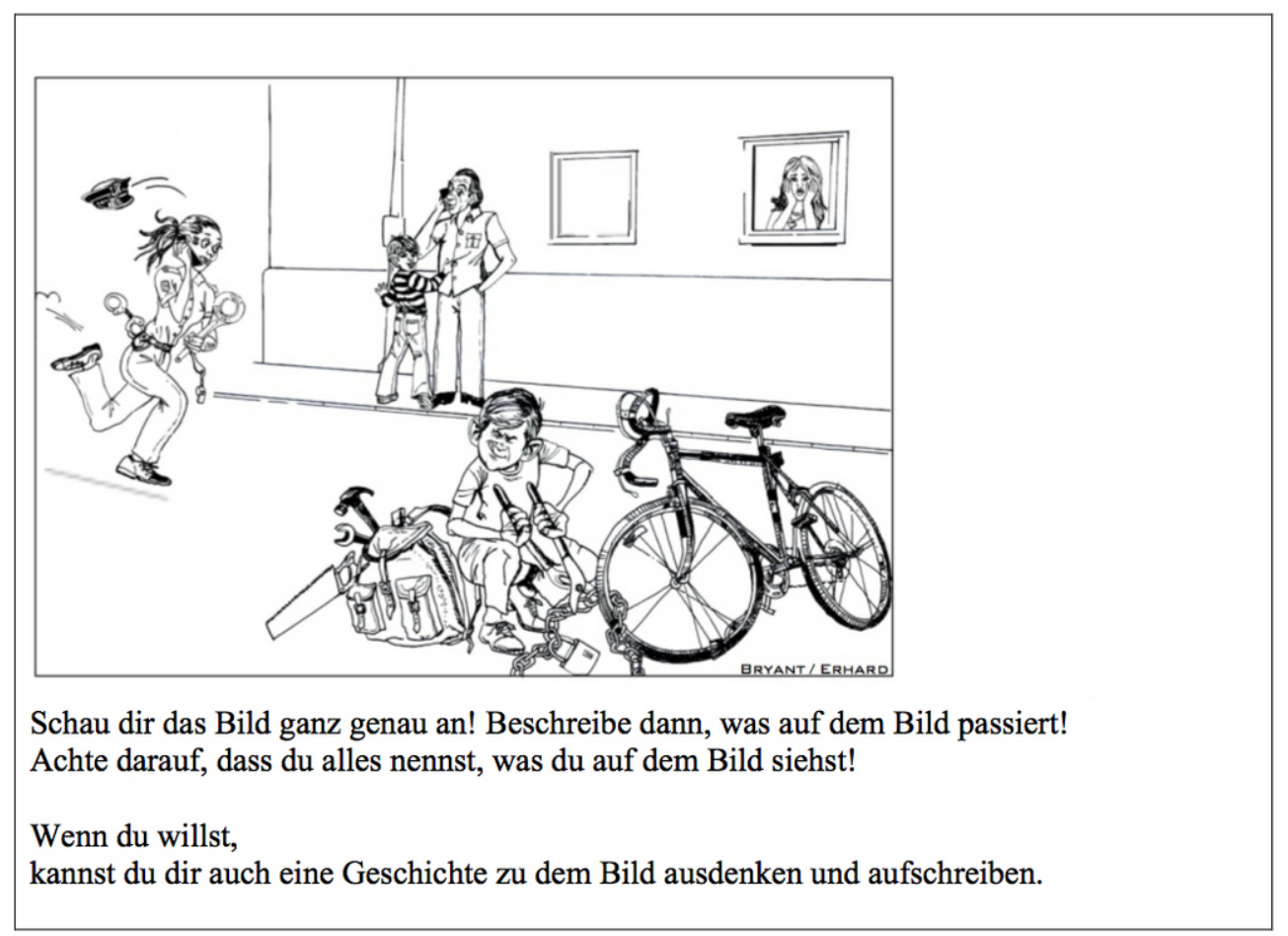

Abbildung 16: Bildvorlage und Aufgabe für schriftliche Sprachprobe (Pre- und Posttest)

Nach den allgemeinen Ausführungen, schauen wir uns nun die drei Sprachaufgaben (schriftlich: Beschreibung eines Bildes/Erzählung, mündlich: Beschreibung einer Bildsequenz/Erzählung, mündlich: Elizitierung statischer Lokalisierungsausdrücke) noch einmal genauer an, und zwar in Kombination mit ersten Ergebnissen. Um Effekte der dramapädagogischen Maßnahme feststellen zu können, eignen sich Bild- und Bildsequenzbeschreibungen in besonderer Weise. Warum? Zur Bewältigung dieser Sprachaufgaben benötigt man die gleichen sprachlichen Mittel wie bei den Inszenierungstechniken ,Standbild' und ,Standbildsequenz', mit denen die Kinder in den Workshops im Rahmen szenischer Improvisationen vertraut gemacht wurden. Dementsprechend könnte man einen aufgabenübergreifenden Transfereffekt erwarten, derart, dass Kinder Strukturen, die sie im Kontext von Standbildern erworben haben, auch auf die Bildbeschreibung übertragen.

Die Ergebnisse der schriftlichen Sprachprobe sollten uns darüber hinaus Auskunft geben, ob medial übergreifende Transfereffekte sichtbar werden. Diagramm 1 dokumentiert diesbezüglich eine beeindruckende Entwicklung. Dargestellt ist der Subordinationsindex in der Voruntersuchung (Pretest) und 
in der Nachuntersuchung (Posttest), und zwar für die drei (in Relation zu den anderen Gruppen) fortgeschrittenen Lernergruppen und für die Kontrollgruppe. Die Mehrheit der Kinder in diesen Gruppen stammen aus den Klassenstufen 4 und 5.

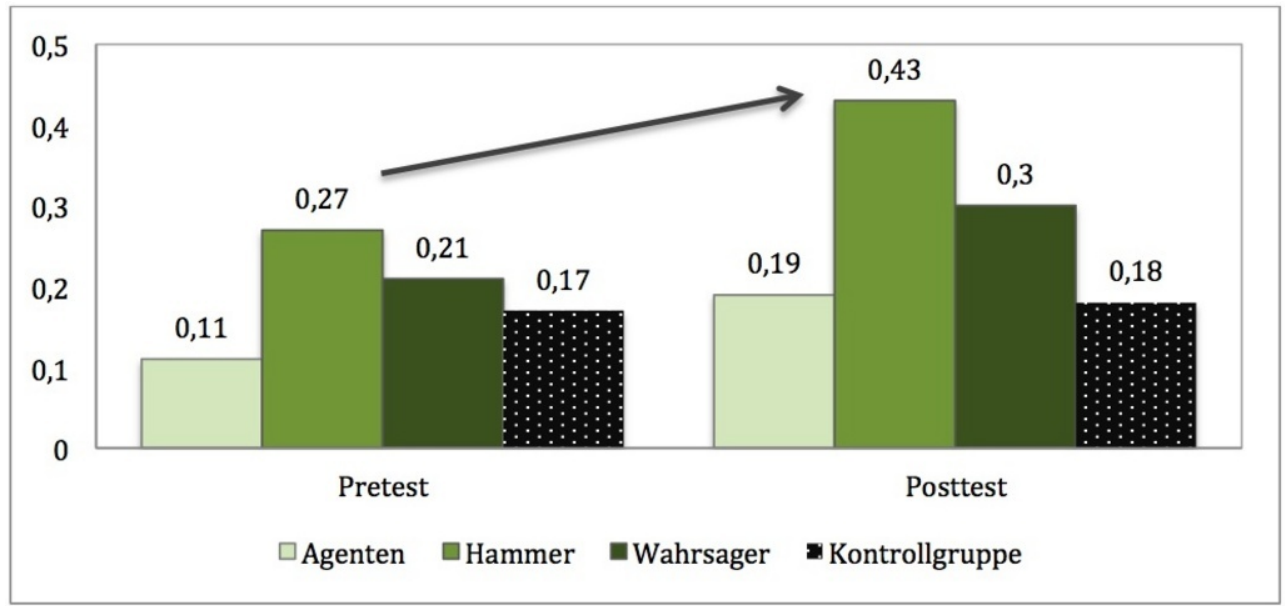

Abbildung 17: Subordinationsindex, schriftliche Sprachprobe (=Diagramm 1)

Der Subordinationsindex wird ermittelt, in dem man die Anzahl der Nebensätze dividiert durch die Anzahl der Gesamtsätze. Ein Subordinationsindex von 0,4 bedeutet beispielsweise, dass auf zehn Gesamtsätze vier Nebensätze kommen. Der von Steinig et al. (2009) auf der Basis von Schüleraufsätzen der Klasse 4 ermittelte Durchschnittsindex beträgt für Kinder mit Deutsch als Muttersprache 0,48 und für Kinder mit Deutsch als Zweitsprache 0,39. Wie in Diagramm 1 anhand der Pretest-Daten zu sehen ist, liegen die Camp-Kinder (mit Sprachförderbedarf) deutlich unter diesen Durchschnittwerten.

Bemerkenswert ist der enorme Zuwachs an Nebensatzkonstruktionen in der Gruppe der Hammer. Im Posttest erzielt diese Gruppe einen Wert, der über dem von Steinig et al. ermittelten Durchschnittswert der DaZ-Population liegt und der sich dem Wert gleichaltriger DaM-Kinder nähert. Wie ist dieser Anstieg zu erklären? Da es sich um die einzige Gruppe handelt, die im Workshop auch den medialen Transfer gezielt unterstützt hat (siehe die Sprachfördereinheit in 3.2) und keine andere Gruppe einen vergleichbaren Anstieg zeigt, ist die Annahme berechtigt, dass diese positive Entwicklung methodisch bedingt ist.

Zoomen wir weiter in den Nebensatzbereich hinein, werden sogar die individuellen Schwerpunkte der einzelnen Workshops sichtbar. So gibt es beispielsweise einen relativ großen Zuwachs an Adverbialsätzen (vgl. Diagramm 2 im Anhang). Bricht man diese Nebensatzkategorie weiter auf, wird erkennbar, welcher Adverbialsatztyp gruppenintern im Fokus stand: bei den Agenten und Wahrsagern Kausalsätze (vgl. Diagramm 3) und bei der Gruppe der Hammer Temporalsätze (vgl. Diagramm 4).

Leider ist in diesem Beitrag nicht der Raum, die Datenmengen ausführlich $\mathrm{zu}$ besprechen, es soll lediglich ein Überblick über die Methoden und 
deren Aussagemöglichkeiten gegeben werden. Damit kommen wir nun zur mündlichen Sprachprobe, die wir vor Ort im Camp erhoben haben. Durch die zeitliche Nähe von Pre- und Posttest erschien es uns hier nicht ratsam, das gleiche Bildmaterial einzusetzen. Es wurde jedoch darauf geachtet, dass die Geschichten von ähnlicher Komplexität sind und dass man auf die drei Akteure mit drei unterschiedlichen Pronomen (er, sie, es) referieren muss (der Junge, die Mutter, das Meerschweinchen vs. der Mann, die Katze, das Eichhörnchen). Die Funktion von Genus, nämlich die Bereitstellung eines pronominalen Sets zur Herstellung referentieller Bezüge, ist für DaZ-Kinder nicht ohne Weiteres einzusehen. Daher haben wir im Camp auf den anaphorischen Gebrauch von Pronomen einen besonderen Schwerpunkt gelegt.
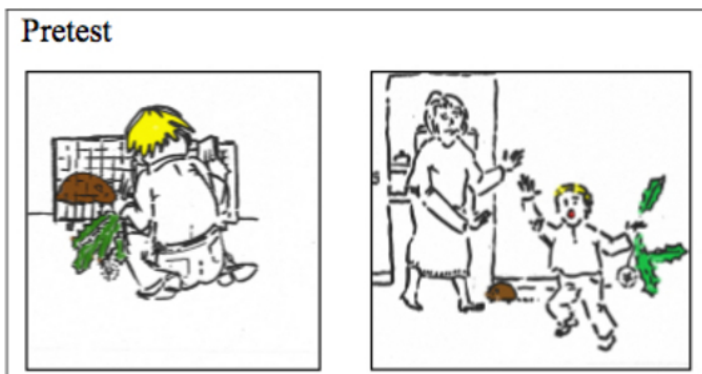

Dies ist eine Bildergeschichte. Schau dir die Bilder der Reihe nach und ganz in Ruhe an, damit du auch gut verstehst, was dort passiert.

(Nachdem das Kind signalisiert, dass es mit der Betrachtung fertig ist:)

Versuche nun diese Bildergeschichte der Eva so gut und spannend wie möglich zu erzählen, damit Eva sie sich gut vorstellen kann, denn Eva sieht die Bilder ja nicht.

Posttest
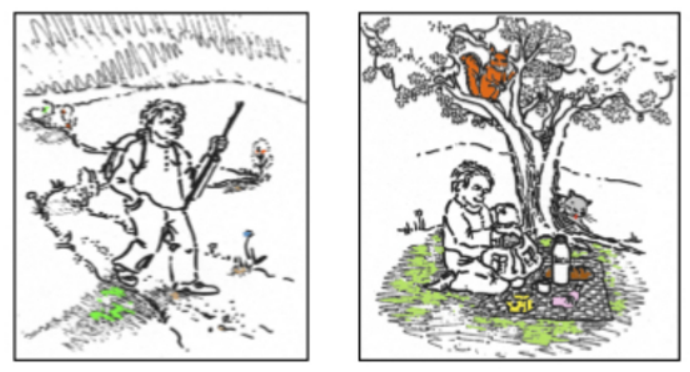

Abbildung 18: Bildvorlagen und Aufgabe für mündliche Sprachproben

Diagramm 5 im Anhang gibt Auskunft über den Gebrauch anaphorischer Pronomen im Pre- und Posttest, und zwar für alle Gruppen. Wie zu erkennen ist, nähern sich die schwächeren Gruppen dem Stand der stärkeren Gruppen an. Sie verzeichnen dementsprechend einen höheren Lernzuwachs in diesem Bereich, wie Diagramm 6 herausstellt.

Auch die individuelle Sprachbetrachtung sollte neben der statistischen Auswertung der Gruppen Berücksichtigung finden, um die Potentiale einer Interventionsmaßnahme auszuloten. Dies gilt umso mehr, wenn die Maßnahme, 
wie im Falle des Pfingstcamps, nur über einen sehr kurzen Zeitraum währt. Beispielsweise verwendete ein Mädchen aus der Gruppe der Hammer im Pretest weder Temporaladverbialsätze noch Komplementsätze - im Posttest hingegen schon, vgl. Abb. 19. Diese Satztypen wurden im Workshop im Kontext der Gerichtsszene eingeführt und geübt.

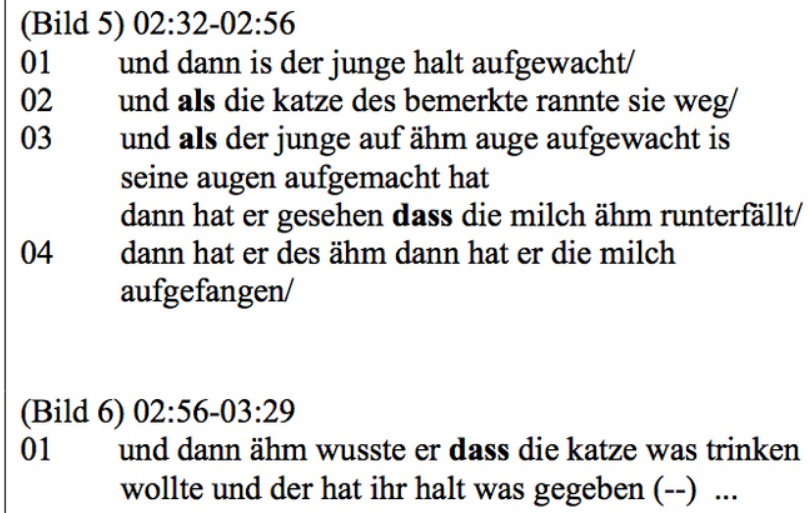

Abbildung 19: Individuelle Sprachbetrachtung, mündliche Sprachprobe

Neben den beiden bislang skizzierten Aufgaben zur (bildgestützten) freien Sprachproduktion (schriftlich vs. mündlich) haben wir noch einen dritten Test angewendet, der im Problembereich der Lokalisierung gezielt Strukturen einfordert, vgl. Abb. 20. Anhand der Äußerungen können wir sehen, wie (un)sicher die Kinder im Gebrauch lokaler Präpositionen, lokaler Verben und in der Verwendung des Dativs sind. Überprüft wurden die Positionsmodi STEHEN, LEHNEN, LIEGEN, HÄNGEN und die Teilraumspezifizierungen IN, AUF, AN, ÜBER, UNTER.

In Diagramm 7 (s. Anhang) ist zunächst nur für den Pretest dargestellt, wie hoch die prozentuale Zielsprachlichkeit in den Gruppen liegt, und zwar in Bezug auf die Verwendung von Präpositionen, Dativ und Positionsverben. $\mathrm{Zu}$ Letzteren ist zu sagen, dass das Deutsche eine Positionsverbsprache ist und Muttersprachler diese Kategorisierung hoch automatisiert vornehmen. Viele DaZ-Kinder, deren Muttersprachen diese Modusspezifizierung nicht realisieren, weichen auf die grammatisch akzeptable Kopulakonstruktion aus ( $x$ ist LOC $y$ ) und unterscheiden sich damit anhaltend von Muttersprachlern (vgl. Bryant 2011).

Wie in Diagramm 7 zu erkennen, treten die Schwierigkeiten insbesondere bei den schwächeren Gruppen auf. In den Workshops dieser Gruppen wurden dementsprechend Lokalisierungsstrukturen stärker einbezogen. Dass dieses Bemühen erfolgreich war, dokumentiert Diagramm 8. Beispielsweise kann die Gruppe der Haie einen beachtlichen Lernzuwachs von $13 \%$ bei den Präpositionen, von $17 \%$ beim Dativ und von $20 \%$ bei den Positionsverben 


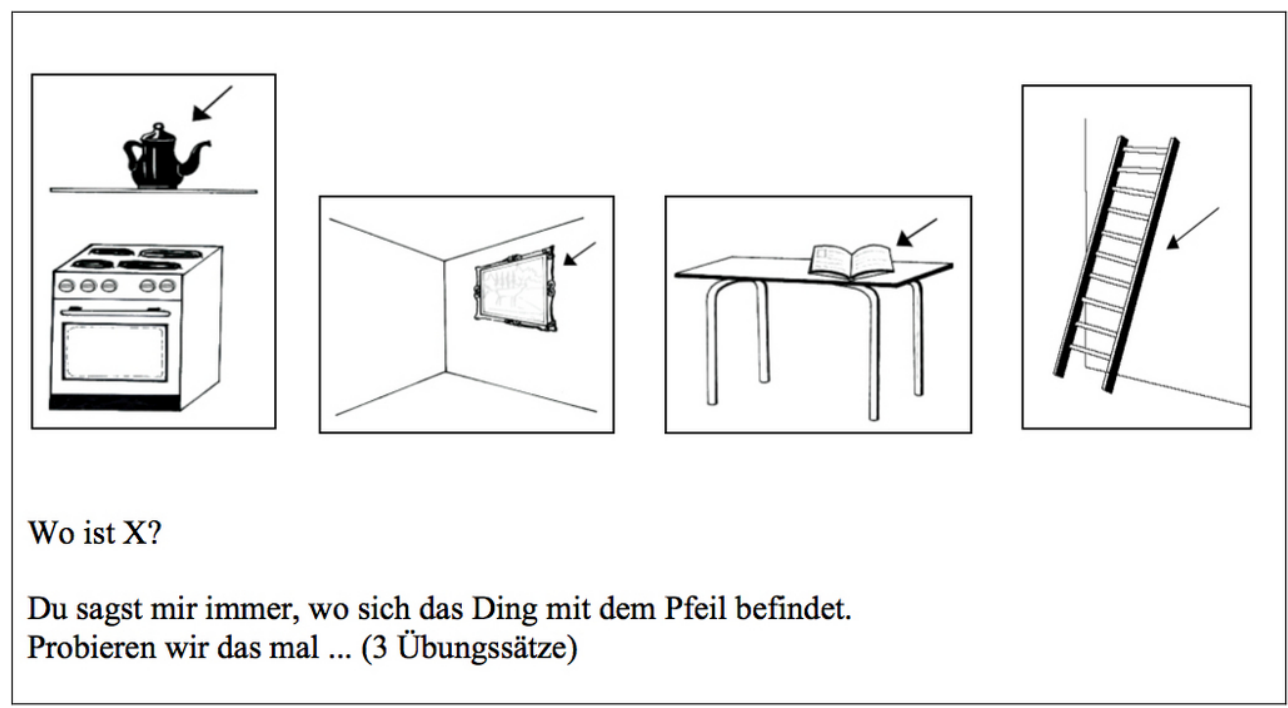

Abbildung 20: Elizitierung statischer Lokalisierungsausdrücke (Bryant 2011, 2012)

verbuchen.

\section{Fazit und Ausblick}

Dargestelltwurde das Tübinger dramapädagogischeFörderkonzept. Die Sprachförderarbeit lanciert eine spezifische registeranhebende Inputanreicherung und bindet Phasen der expliziten Grammatikvermittlung und der Sprachreflexion in den dramapädagogischen Prozess ein. Die positiven Ergebnisse, die trotz der Kürze der Intervention bereits erreicht wurden, stimmen uns optimistisch, dass wir mit der Konzeption auf dem richtigen Weg sind. Um die Nachhaltigkeit zu sichern, wäre darüber nachzudenken, ob und wie man ausgehend von einem impulsgebenden und die Kinder begeisternden Ferienprojekt die dramapädagogische Arbeit an der Schule fortsetzt. Damit dieser Schritt für die gut ausgebildeten und nun auch praxiserprobten Studierenden möglich wird, müssen die curricularen Voraussetzungen geschaffen werden. Der Tübinger DaZ-Studiengang sieht beispielsweise ein einjähriges studienbegleitendes Praktikum vor. Wünschenswert wäre eine solche Option auch für das Lehramt. Die Studierenden könnten sich in einer Theater-AG oder auch im Deutschunterricht im Tandem mit dem Fachlehrer / der Fachlehrerin als Experten auf dem Gebiet der dramagrammatischen Sprachförderung einbringen.

\section{Bibliografie}

Bryant, Doreen (2011): Der Erwerb von Positionsverben - Warum Kinder so an ,hängen' hängen? In: Linguistische Berichte 226, 199-242 
Bryant, Doreen (2012): Lokalisierungsausdrücke im Erst- und Zweitspracherwerb. Typologische, ontogenetische und kognitionspsychologische Überlegungen zur Sprachförderung in DaZ. Baltmannsweiler: Schneider Verlag Hohengehren

Bryant, Doreen; Azizpur, Pegah; Bohnet, Eva; Susanne Göttfert; Andrea Heldmaier; Laura Hermenau; Lehmann, David; Michaela Neichel; Nadine Noschka; Marco Petering (in Vorb.): Das Tübinger Sprachförderkonzept: Explizite Sprachvermittlung im dramapädagogischen Kontext für Grundschulkinder mit DaZ. Sprachfördereinheiten mit Anleitung für LehrerInnen und ErzieherInnen. Universität Tübingen

Bybee, Joan (2008): Usage-based grammar and second language acquisition. In: Robinson, Peter; Ellis, Nick (eds.): Handbook of Cognitive Linguistics and Second Language Acquisition. New York: Routledge, 216-236

Casenhiser, Devin; Goldberg, Adele E. (2005): Fast mapping between a phrasal form and meaning. In: Developmental Science 8, 500-508

Dudenredaktion (2005): DUDEN. Die Grammatik. Mannheim: Bibliographisches Institut \& F.A. Brockhaus AG

Even, Susanne (2003): Drama Grammatik. Dramapädagogische Ansätze für den Grammatikunterricht Deutsch als Fremdsprache. München: Iudicium

Gogolin, Ingrid; Roth, Hans-Joachim (2007): Bilinguale Grundschule: Ein Beitrag zur Förderung der Mehrsprachigkeit. In: Anstatt, Tanja (ed.): Mehrsprachigkeit bei Kindern und Erwachsenen. Erwerb - Formen Förderung. Tübingen: Attempto Verlag, 31-45

Haberzettl, Stefanie (2009): Förderziel: Komplexe Grammatik. In: LiLi 153, 80-95

Kniffka, Gabriele; Siebert-Ott, Gesa (2007): Deutsch als Zweitsprache. Lehren und Lernen. Paderborn et al.: Schöningh

Koch, Peter; Oesterreicher, Wulf (1994): Schriftlichkeit und Sprache. In: Günther, Hartmut; Ludwig, Otto (eds.): Schrift und Schriftlichkeit. Ein interdisziplinäres Handbuch, 1. Halbband, Berlin: de Gruyter, 587-604

Rösch, Heidi (2007): Das Jacobs-Sommercamp - neue Ansätze zur Förderung von Deutsch als Zweitsprache. In: Bernt Ahrenholz (ed.): Kinder mit Migrationshintergrund. Spracherwerb und Fördermöglichkeiten. Freiburg: Fillibach, 287-302

Schewe (1993): Fremdsprache inszenieren. Zur Fundierung einer dramapädagogischen Lehr- und Lernpraxis. Oldenburg: Didaktisches Zentrum, Carl von Ossietzky-Universität Oldenburg

Schmölzer-Eibinger, Sabine (2011 $\left.{ }^{2}\right)$ : Lernen in der Zweitsprache. Grundlagen und Verfahren der Förderung von Textkompetenz in mehrsprachigen Klassen. Tübingen: Narr Verlag

Siebert-Ott, Gesa (2001): Frühe Mehrsprachigkeit. Probleme des Grammatikerwerbs in multilingualen und multikulturellen Kontexten. Tübingen: Niemeyer 
Stanat, Petra; Baumert, Jürgen; Müller, Andrea G. (2005): Förderung von deutschen Sprachkompetenzen bei Kindern aus zugewanderten und sozial benachteiligten Familien. In: Zeitschrift für Pädagogik 31, 856-875

Steinig, Wolfgang; Betzel, Dirk; Geider, Frank Josef; Herbold, Andreas (2009): Schreiben von Kindern im diachronen Vergleich. Münster et al.: Waxmann

Tselikas, Elektra I. (1999): Dramapädagogik im Sprachunterricht. Zürich: Orell Füssli

Wegener, Heide (1995): Die Nominalflexion des Deutschen, verstanden als Lerngegenstand. Tübingen: Niemeyer

Danksagung —Dank des außerordentlichen Einsatzes aller Beteiligten wurde das Pfingstcamp zu einem erfolgreichen Pilotprojekt für die Tübinger Community. Die Projektbeteiligten in alphabetischer Reihenfolge: Patric Altwig, Pegah Azizpur, Andrea Batzel, Eva Bohnet, Thorsten Bohl, Doreen Bryant, Hans Ignaz Dieter, Sirka Elfert, Christine Faulstich, Gladys Nunez Gonzalez, Susanne Göttfert, Judith Halisch, Andrea Heldmaier, Laura Hermenau, Christian Hoh, Mehtap Ince, Marie Kaiser, Elvira Kazeva, David Lehmann, Marije Michel, Michaela Neichel, Nadine Noschka, Susanne Omran, Marco Petering, Volker Schubert, Michael Spaun, Anne Vogt, Birgit Walker, Christine Wetzig, Julia Will.

Danke an Birla Erhard und Achim Wetzig für die Zeichnungen, an das MPI in Nijmegen für die Erlaubnis, Auszüge aus dem TRPS-Booklet nutzen zu dürfen, an Günther Koch und Hans-Jörg Schabel für die Fotos und an Maik Walter für den inspirierenden Austausch und die beflügelnde Lehre in Tübingen.

Eva Bohnet, Susanne Göttfert, Michaela Neichel, Nadine Noschka und Heike Bischoff sei herzlichst gedankt für ihre Unterstützung bei der Transkription und Datenaufbereitung, Mehtap Ince für das Einholen der Schüleraufsätze und Nadine Noschka und Michaela Neichel für die Datenerhebung der Kontrollgruppen.

Das Tübinger Theaterprojekt wurde gefördert durch die BOSCH-Stiftung. 


\section{Anhang}

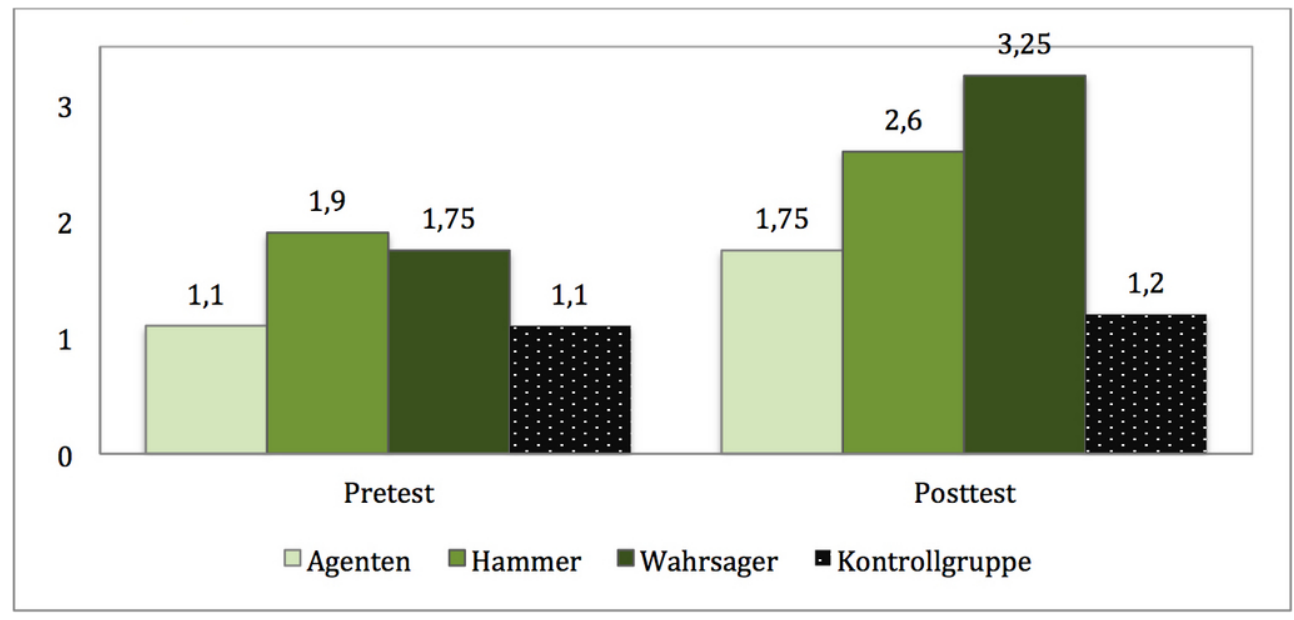

Abbildung 21: Adverbialsätze (Mittelwert), schriftliche Sprachprobe

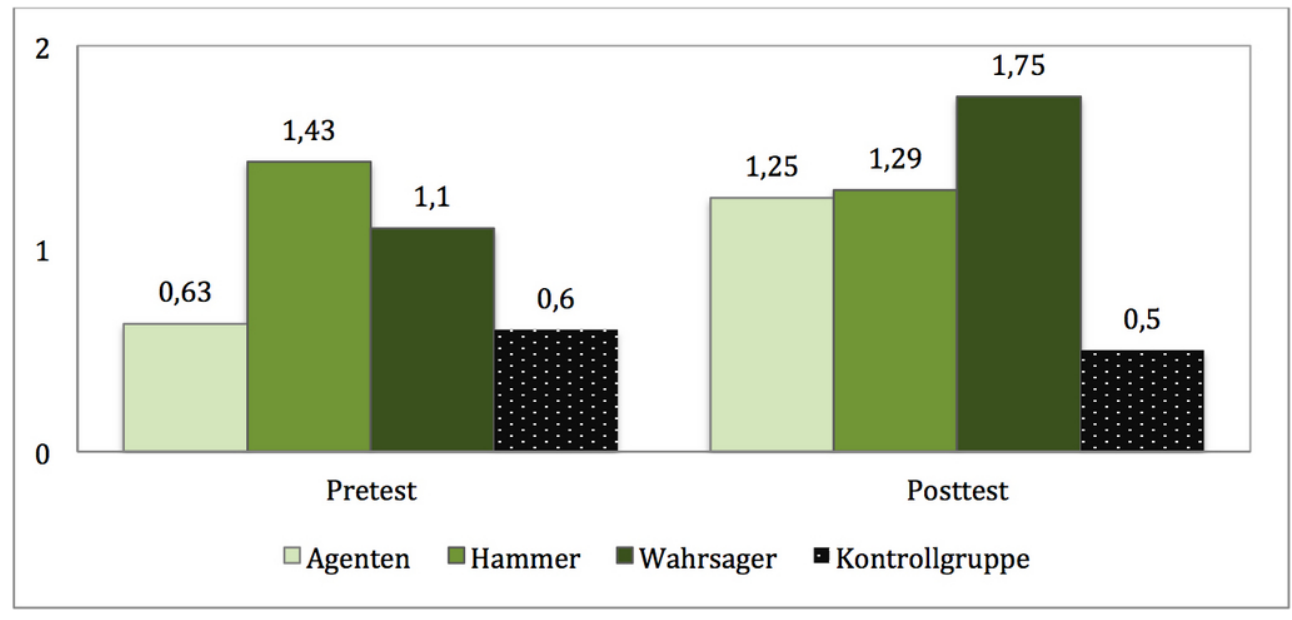

Abbildung 22: Adverbialsätze: Kausalsätze (Mittelwert), schriftliche Sprachprobe 


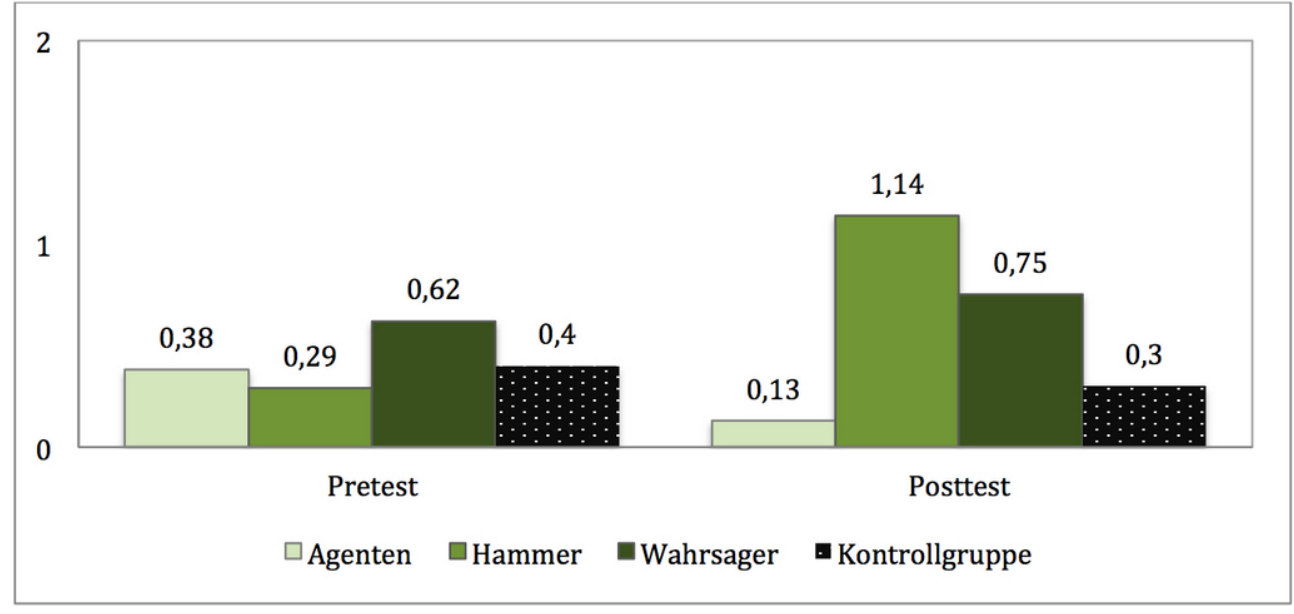

Abbildung 23: Adverbialsätze: Temporalsätze (Mittelwert), schriftliche Sprachprobe

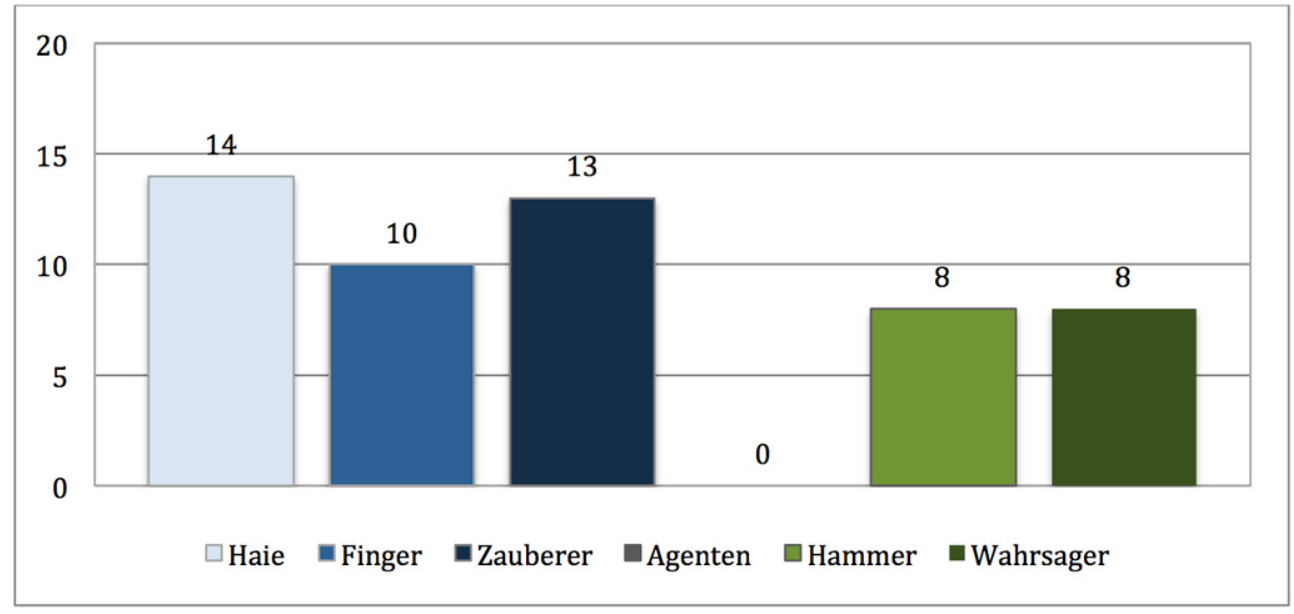

Abbildung 24: Anaphorische Pronomen, Lernzuwachs in \% 


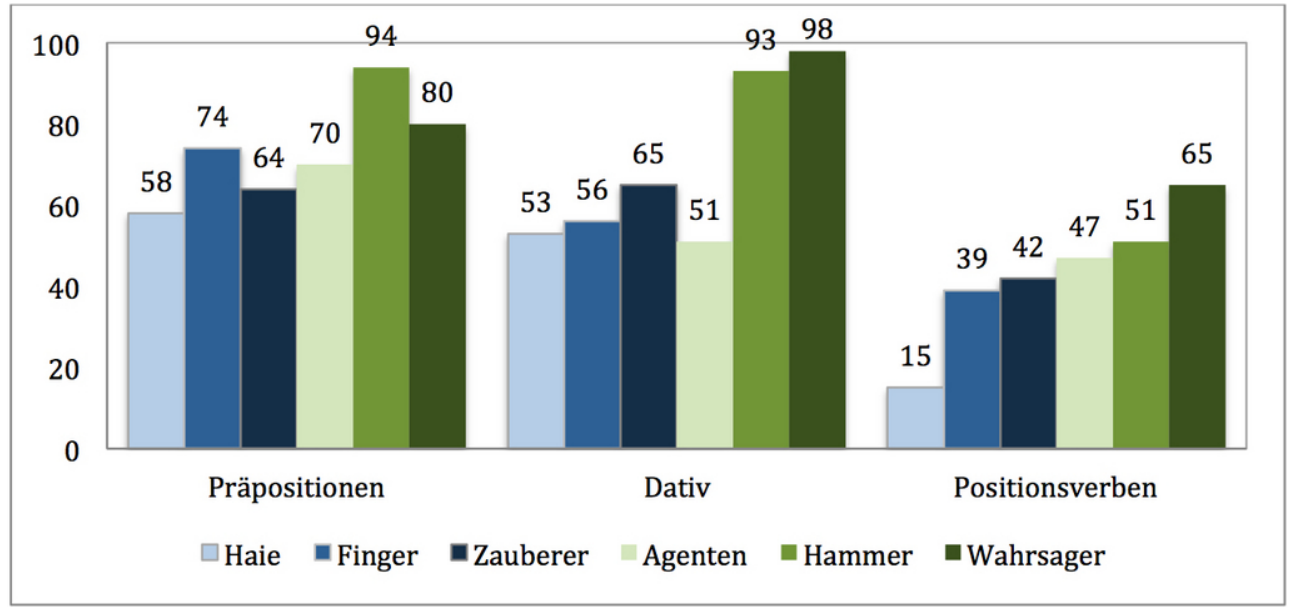

Abbildung 25: Statische Lokalisierung, Zielsprachlichkeit in \% - Pretest

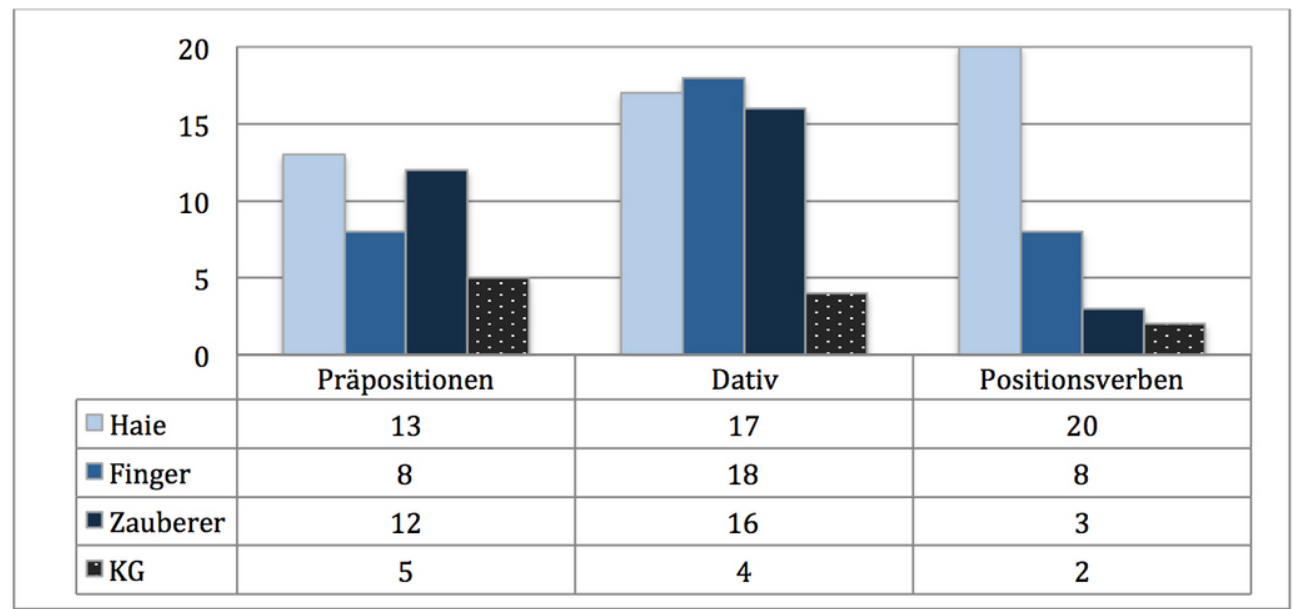

Abbildung 26: Statische Lokalisierung, Lernzuwachs in \% 